\title{
Understanding the Experiences of Remote Workers: Opportunities for Ambient Workspaces at Home
}

\author{
Eleni Kallopi Margariti *, Ridita Ali, Remco Benthem de Grave, David Verweij, Jan Smeddinck \\ and David Kirk
}

Open Lab, School of Computing, Newcastle University, Newcastle Upon Tyne, United Kingdom

As the COVID-19 pandemic has forced many to work remotely from home, collaborating solely through digital technologies, a growing population of remote home workers are faced with profound wellbeing challenges. Passive sensing devices and ambient feedback have great potential to support the wellbeing of the remote workers, but there is a lack of background and understanding of the domestic workplace in terms of physical and affective dimensions and challenges to wellbeing. There are profound research gaps on wellbeing in the domestic workplace, with the current push for remote home and hybrid working making this topic timely. To address these gaps and shape a starting point for an

OPEN ACCESS

Edited by:

John Alexander Waterworth Umeå University, Sweden

Reviewed by: Galena Pisoni, University of Nice Sophia Antipolis,

France

Arminda Guerra,

University of Lisbon, Portugal

*Correspondence:

Eleni Kallopi Margariti

E.K.Margariti2@newcastle.ac.uk

Specialty section: This article was submitted to

Human-Media Interaction,

a section of the journal Frontiers in Computer Science

Received: 27 February 2021

Accepted: 04 August 2021 Published: 03 September 2021

Citation:

Margariti EK, Ali R, Benthem de Grave $R$, Verweij $D$, Smeddinck $J$ and Kirk D (2021) Understanding the Experiences of Remote Workers: Opportunities for

Ambient Workspaces at Home.

Front. Comput. Sci. 3:673585. doi: 10.3389/fcomp.2021.673585 "ambient workspaces" agenda, we conducted an exploratory study to map physical and affective aspects of working from home. The study involved both qualitative and quantitative measures of occupant experience, including sensor wristbands, and a custom web application for self-reporting mood and aspects of the environment. It included 13 participants for a period of 4 weeks, during a period of exclusive home working. Based on quantitative and qualitative analysis, our study addresses wellbeing challenges of the domestic workplace, establishes correlations between mood and physical aspects, and discusses the impact of feedback mechanisms in the domestic workplace on the behavior of remote workers. Insights from these observations are then used to inform a future design agenda for ambient technologies that supports the wellbeing of remote workers; addressing the design opportunities for ambient interventions in domestic workspaces. This work offers three contributions: 1) qualitatively and quantitatively informed understandings of the experiences of homeworkers; 2) a future design agenda for "ambient home workspaces"; and 3) we propose three design concepts for ambient feedback and human-Al interactions in the built environment, to illustrate the utility of the design agenda.

Keywords: wellbeing, remote-worker, ambient technology, adaptive architecture, wearable feedback

\section{INTRODUCTION}

Over the last 3 decades there has been increasing interest in supporting more flexible working, which has seen the rise of "remote workers," who spend increasing amounts of time working from home "offices" (Fan Ng, 2010). This has particularly come to the fore during the global COVID-19 pandemic, which has both forced people to work from home, but also increased discussion of the possible values of home-working, post-pandemic (Khazan, 2020). The majority of past research on 
workplaces focuses on office buildings (Fortmann et al., 2013; Mark et al., 2015; Aryal et al., 2018; Alavi et al., 2018b; Clear et al., 2018; Lee et al., 2021). There have been extensive studies concerning the potential role of digital technology in supporting the well-being of office workers (see for example, Ludden and Meekhof, 2016). Recently there has been an emerging academic discourse around Human-Building Interaction (HBI) (Alavi et al., 2016; Alavi et al., 2018a), and some of this crosses-over into discussion of workplaces and the wellbeing of occupants (Schnädelbach and Kirk, 2019). However, this leaves prominent research gaps in understanding people's engagement with physical and ambient aspects of the lived domestic workplace, the challenges they raise for affective and physical wellbeing of remote workers, and the opportunities for diverse digital feedback mechanisms-such as wearable mediated feedback- to support them. Collecting such data can provide the grounds for designing novel ambient technologies to support the wellbeing of remote workers under the term "ambient homeworkspaces," drawing upon and contributing to the research fields of Ambient (Intelligent) Spaces (Pousman and Stasko, 2006; Bakker et al., 2016; Yannoudes, 2016), HBI (Dalton, 2016; Jäger, 2017; Nembrini et al., 2017; Schnädelbach and Kirk, 2019) and Human-AI interaction (Alavi et al., 2016; Alavi et al., 2020) within the built environment.

To address these research gaps and provide a starting point for shaping a design agenda for "ambient home-workspaces," we conducted an exploratory study to map physical and affective aspects of working from home. The study involved both qualitative and quantitative measures of occupant experience, including sensor wristbands, and a custom web application for self-reporting mood and aspects of the environment with 13 participants for a period of 4 weeks (during a period of exclusive home-working). Based on the analysis of both the quantitative and qualitative data, the study addresses the wellbeing challenges of the domestic workplace, establishes correlations between mood and physical aspects, and discusses the impact of feedback mechanisms in the domestic workplace on the behavior of remote workers. Insights from these observations are then used to inform a future design agenda for ambient technologies that supports the wellbeing of remote workers; addressing the design opportunities for ambient interventions in domestic workspaces.

Research questions addressed in this study include:

RQs

1) How do remote workers manipulate their physical spaces to create a workplace?

2) What are the challenges remote workers experience in their domestic workspaces?

3) How do aspects of their surroundings impact on remote workers' embodied wellbeing?

4) What are the potentials of wearable-mediated and moodreflective feedback to support remote workers' wellbeing?

This paper contributes 1) a detailed understanding (both qualitative and quantitative) of the experiences of homeworkers; 2) a future design agenda for "ambient home workspaces"; and 3) three design concepts for ambient feedback and human-AI interactions in the built environment, to illustrate the utility of the design agenda.

Below we begin by sketching out some areas of relevant literature, before turning to articulate our methodological approach to the research. Then we present the findings of the field study before discussing them in relation to a proposed design agenda, and the generated concept designs.

\section{The Remote Worker and Wellbeing Challenges}

Lockdowns initiated during the COVID-19 pandemic have established the home as a permanent workspace for many (Khazan, 2020). The domestic spaces in which this remote work is conducted are generally limited and congested (Fan Ng, 2010). The remote home worker is thereby physically based in a highly unregulated and heterogenous domestic space-a space for which separation of work and home is a challenge (Stawarz et al., 2013; Toch et al., 2020). Remote workplace collaboration occurs predominantly through technological media; with all social activities reduced to two-dimensional screens (Deloitte LLP, 2018). This raises challenges of remote home workers feeling both isolated and yet constantly connected (Deloitte LLP, 2018), struggling to keep a balance between a domestic and technology mediated work-life (Toch et al., 2020).

Digital transformations have contributed to eliminating physical movement in the workplace (Choi et al., 2016; Cambo et al., 2017; Brombacher et al., 2020; Damen et al., 2020) through enhancing connectivity between remote workers (Haliburton and Schmidt, 2020), and intensifying technologymediated collaboration (Deloitte LLP, 2018; Haliburton and Schmidt, 2020). The use of such collaborating technologies does not mediate the benefits of informal, face-to-face interaction, as well as physical activity for the wellbeing of the remote workers (Haliburton and Schmidt, 2020). Lack of physical activity has resulted in ongoing health problems amongst connected remote workers, including chronic mental health issues (Chodan et al., 2019) and physiological problems (Haliburton and Schmidt, 2020). Past work in HCI has outlined some of these issues with a particular emphasis on work stress (Alonso et al., 2008; Chodan et al., 2019), low productivity and motivation (Mark et al., 2015; Epstein et al., 2016), leaving the home office broadly unexplored.

Within this context, the argument for ambient technologies to support the remote home workers falls into place. Ambient systems have been previously used to mitigate "always-on" environments (Stawarz et al., 2013; Toch et al., 2020), and to support mental and physical wellbeing in various domestic and health applications (Weiser and Brown, 1995; Ludden and Meekhof, 2016; Bittner et al., 2019; Wohn et al., 2020).

\section{Introducing Ambient Workspaces}

Advances in sensory and computing power in the buildings are giving rise to increasing opportunities for ambient spaces (Schnädelbach and Kirk, 2019). Ambient systems connected to data sources can communicate information in an aesthetically pleasing and calm manner (Weiser and Brown, 1995; Pousman and Stasko, 2006; Ludden and Meekhof, 2016), seamlessly 
merging with the surrounding space (Bakker et al., 2016). Highly relevant to workplace needs, various types of ambient systems (Weiser and Brown, 1995; Ludden and Meekhof, 2016) can present information in the periphery without distracting or burdening the users, helping in prioritisation and cognitive easing (Pousman and Stasko, 2006; Caraban et al., 2019). Examples of ambient systems include adaptive architectures - subtle changes in an architectural space (Schnädelbach et al., 2012; Dalton, 2016; Yannoudes, 2016; Nabil et al., 2017a,b; Jäger, 2017; Bader et al., 2019; Schnädelbach and Kirk, 2019), connected sensing objects -IoT (Bittner et al., 2019), or light feedback (Fortmann et al., 2013; Davis et al., 2016; Pereira et al., 2016). Focusing on ambient technology research for the workplace, we briefly highlight examples from HBI, $\mathrm{HCI}$ and Ubicomp literature. We broadly outline key projects that address aspects of wellbeing ${ }^{1}$ in workspaces (both office and domestic) such as the physical environment, comfort, social interaction, physical activity, mood, and stress.

A breadth of research has established relationships between physical and environmental factors, and the wellbeing and productivity of employees in office spaces using sensory devices (Alavi et al., 2017; Snow et al., 2017; Aryal et al., 2018; Clear et al., 2018; Constantinides et al., 2020; Lee et al., 2021). Some works study the impact of static spatial features on social interactions and social behavior in the workplace (Mark et al., 2014; Mark et al., 2015; Alavi et al., 2018b). A few projects use sensory devices to study informal human interactions and socio-spatial comfort (Lee et al., 2021) within office spaces, and to inform the future design of workspaces (Alavi et al., 2017; Alavi et al., 2020).

More recently, a growing body of work is concerned with indoor climate in the office spaces (Alavi et al., 2017; Aryal et al., 2018; Constantinides et al., 2020), it's impact on productivity and wellbeing, and the most appropriate ways to sense, communicate and engage the building occupants in co-shaping it (Snow et al., 2017; Clear et al., 2018; Lee et al., 2021). Alavi et al. (2017) addressed the multidimensional issue of comfort in the workplace through Comfortbox - a sensing physical display of environmental data, engaging the office space occupants in gaining awareness on their embodied comfort. Equally, air quality has been the focus of recent HCI and Human-AI Interaction research (Snow et al., 2016; Dang et al., 2020; Kim and Li, 2020; Zhong et al., 2020). Hilo (Zhong et al., 2020), a predictive system for CO2 mediated by wearable feedback, notifies the occupants of an office space to open the windows when indoor quality reaches a critical point, given the outdoor pollution is low. The work of Kim and Li (2020) addresses ambient light as air quality feedback and its perceivability as eco feedback. Dang et al. (2020) explore different forms of ambient air quality notifications for smart rooms, addressing user experiences and expectations of indoor air quality visualizations.

Light has been the most explored medium for ambient interactions (Rogers et al., 2010; Fortmann et al., 2013; Snyder et al., 2015; Pereira et al., 2016; Yu et al., 2018; Zhang et al., 2020). Many projects are coupling light with feedback to promote

\footnotetext{
${ }^{1}$ See PROWELL Key Performance Indicators (KPIs) of wellbeing at the physical workplace at https://www.innovativeworkplaceinstitute.org/workplace-wellbeingprowell.php.
}

movement and physical activity in the workplace (Rogers et al., 2010; Fortmann et al., 2013; Pereira et al., 2016). Light as biofeedback (generated by wearable sensors measuring arousal levels) was used to explore the implications of mood awareness in the workplace-see "MoodLight" (Snyder et al., 2015). "DeLight" explores the potential of Philips Hue to support de-stressing, relaxing, and encouraging better breathing patterns, albeit in a laboratory set-up (Yu et al., 2018).

Other projects explore the potential of adaptive and digitally enhanced architectural elements to encourage social interaction at the workspace (Follmer et al., 2013; Takashima et al., 2016; Grønbæk et al., 2017; Jäger et al., 2017; Grønbæk et al., 2020; Petersen et al., 2020). Working on dynamic affordances and proxemic transitions, Grønbæk et al. (2017), Grønbæk et al. (2020) and Petersen et al. (2020) explore the potentials of shape changing interfaces to support social interactions in collocated informal meetings. Kirigami table (Grønbæk et al., 2020 ) is a shape changing meeting table and an interactive display for co-located collaboration, creating opportunities for dynamic socio-spatial interactions. A different approach by SenseSeat (Campos et al., 2018), a computational multisensorial seat that can be digitally controlled and vary the frequency and intensity of visual, auditory, and olfactory stimulus; creating furniture that enable control of the ambient surroundings for personalized comfort.

Augmenting information on static architectural elements through AR and projection technologies has been the focus of several works (Huber, 2014; Perteneder et al., 2016; Lee et al., 2019). Examples are projects on augmented barriers for blocking distractions (Lee et al., 2019), or office lamps that extend display in the periphery, allowing desk and wall surfaces to work as ambient work surfaces (Huber, 2014; Winkler et al., 2014; Lee et al., 2019). LumenAR (Huber, 2014) and FACT (Huber, 2014) are projects that explore potentials of portable $\mathrm{AR}$ projection to assist with work tasks while extending the display. Projection can also work in large displays (Pousman and Stasko, 2006; Perteneder et al., 2016; Petford et al., 2016; Zhao et al., 2017); ASPECTA Toolkit (Petford et al., 2016) explores low-cost ways to create large projected displays that adapt to any room.

Passive data collection in the built environment through smart phones, wearables and embedded sensors is broadly framed within the ambient spaces' research agenda; with projects employing such technologies to monitor mood (Church et al., 2010; Mark et al., 2014; Hänsel, 2016; Campos et al., 2018), stress (Alonso et al., 2008; Kikhia and Hallberg, 2013; Yu et al., 2018; Chodan et al., 2019) and physical activity of the building occupants (Gouveia et al., 2016; Cambo et al., 2017). The SEBA system (Chodan et al., 2019) is an example of an approach for monitoring psychological stress continuously at the workplace using wearable sensors. Some of these projects have been criticized for the use of sensitive data in the workplace (Chodan et al., 2019) and the fact that they often fail to proactively engage the users in utilizing their data for their own benefit through appropriate feedback mechanisms (Rogers et al., 2010). The importance of feedback on wellbeing at the workplace has been addressed by several studies, looking at deploying persuasive technologies for behavior change at the 
office (Rogers et al., 2010; Ludden and Meekhof, 2016). Finally, a few studies explored the impact of mood self- tracking and sharing on the individual and collective wellbeing in the lived workspace (Church et al., 2010; Mora et al., 2011; Meyer et al., 2017), albeit without addressing ambient feedback in that context.

\section{Research Opportunities for the Domestic Workplace}

With past literature focusing on conventional office spaces (Alavi et al., 2017; Alavi et al., 2018b), productivity or stress (Alonso et al., 2008; Aryal et al., 2018; Clear et al., 2018; Chodan et al., 2019); there are many potentials for ambient technology in the domestic workplace. The main contribution of this work is to establish knowledge of how remote home workers engage with the workplace at home, what aspects are important for their wellbeing, and what types of ambient feedback would be more appropriate for supporting them.

New directions in ambient spaces research emphasize on engaging the occupants through wearable-mediated feedback (Zhong et al., 2020) and address contextual privacy through prioritizing passive data collection (Aryal et al., 2018). Recommendations and predictions of behavior of the building occupants (Human -AI interaction) are discussed in a small number of recent works (Alavi et al., 2016; Zhong et al., 2020), showing much potential while raising ethical considerations. As a secondary contribution, this work takes these developments into account, alongside the results of our empirical work, to frame a design agenda for ambient workspaces at home-addressing future remote and hybrid workplace models with an emphasis on wellbeing.

\section{MATERIALS AND METHODS}

\section{Study Set Up}

The study engaged 13 participants for a period of 4 weeks during June-July-August 2020. The participants were posted a wearable band (Mi Band 2) measuring type of physical activity, step count, and heart rate data. They were asked to wear the band during the whole study period-preferably 24/7, or at least during their working hours. The participants also received a daily email with a link to a purpose-built static web app (see Figure 1. The app offered an easy-to-use interface for documenting aspects of mood and to characterize the individual workplaces using sliders, text input fields (diary entries) and image uploads. The participants were asked to upload a picture of their workplace, evaluate their surroundings and their mood using sliders, and fill in a short diary for that day. The email was sent either in the midday break (at 12:00) or at the end of workday (at 17:00) in alternate; aiming to create a pleasant "routinely variation" that the participants expected without interrupting them in their work. Depending on the time sent, dedicated questions were asked via the app to trigger either "on the spot" awareness-i.e., where are you working now? how do you feel now?-or self-reflection of participants-i.e., how has your workday been? Besides their daily feedback via the app, participants provided the researchers with the wearable's data-heart rate, physical activity (steps and distance), sleep - at the end of the 4-week study period. Participants were also interviewed (30' semi-structured interviews via zoom) after the end of their enrollment, to reflect on their overall experience of the study and the technologies used.

\section{Participants}

The 13 participants (see Table 1) were recruited via social media (Twitter, Facebook). Their educational background varied, coming from research, software, design, and psychology sectors. In terms of their educational level, the participants ranged from BSc to Post Doc. Their previous experience working from home differs -which is acknowledged, together with information on their domestic and home workspace set up.

\section{Data Collection: Data and Technologies}

The study was conducted using commodity wearable bands Mi band 2 and a purpose-built static web app (hosted in GitHub

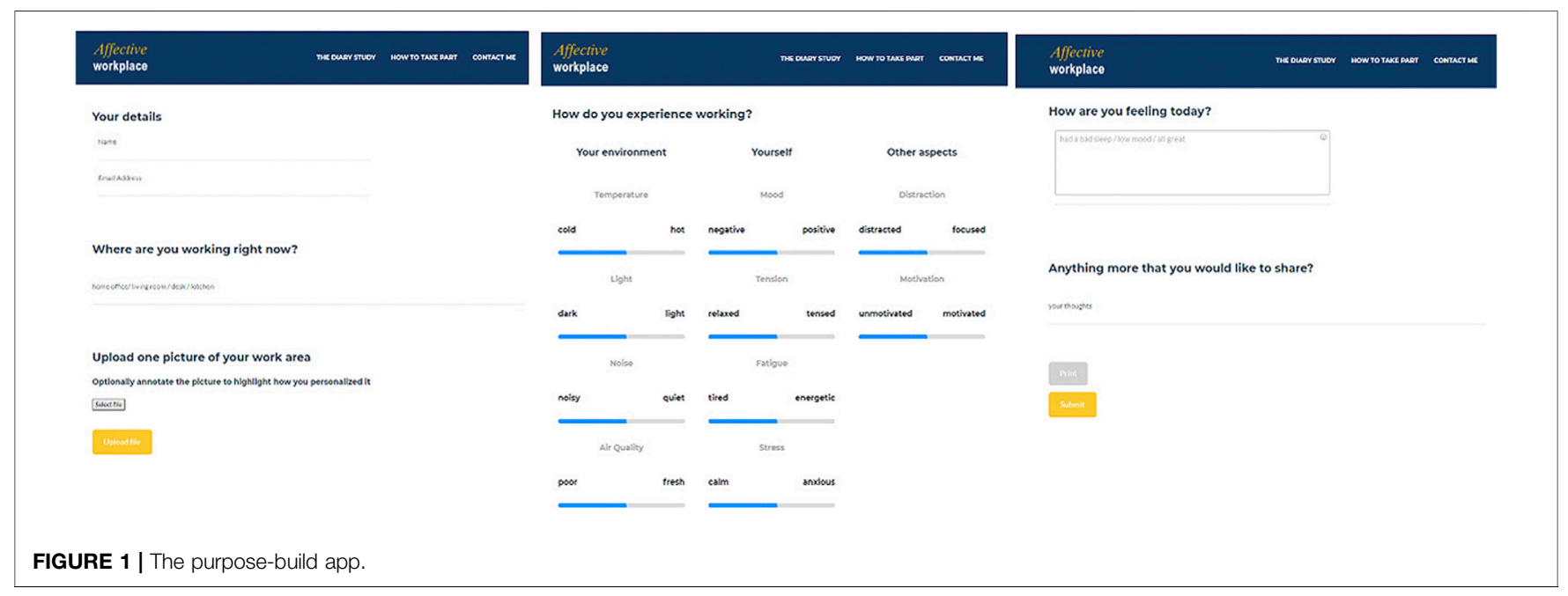


TABLE 1 | Participants.

\begin{tabular}{|c|c|c|c|c|c|c|c|}
\hline $\begin{array}{l}\text { Participant } \\
\text { number }\end{array}$ & Age & Gender & $\begin{array}{l}\text { Level of } \\
\text { education }\end{array}$ & Occupation & $\begin{array}{l}\text { Previously } \\
\text { working from home }\end{array}$ & Health conditions & Domestic set up \\
\hline P01 & 55 & $M$ & MSc & Researcher & $\begin{array}{l}8 \text { years remote work as } \\
\text { an IT consultant }\end{array}$ & N/A & $\begin{array}{l}\text { Lives alone in a flat, has a separate } \\
\text { room for work office }\end{array}$ \\
\hline P02 & 31 & $\mathrm{~F}$ & PhD & Researcher & 2 years occasionally & N/A & $\begin{array}{l}\text { Lives with partner at a house, } \\
\text { shared home office }\end{array}$ \\
\hline P03 & 27 & $\mathrm{~F}$ & $\mathrm{MSc}$ & $\begin{array}{l}\text { Interaction } \\
\text { designer }\end{array}$ & None & N/A & $\begin{array}{l}\text { Lives with partner at a flat, shared } \\
\text { home office }\end{array}$ \\
\hline $\mathrm{PO4}$ & 32 & $\mathrm{~F}$ & $\mathrm{MSc}$ & Manager & None & $\begin{array}{l}\text { Post-traumatic stress } \\
\text { disorder }\end{array}$ & $\begin{array}{l}\text { Lives with partner at a house, } \\
\text { separate home office }\end{array}$ \\
\hline P05 & 36 & $\mathrm{~F}$ & MSc & $\begin{array}{l}\text { Software } \\
\text { engineer }\end{array}$ & 3 years remote engineer & Endometriosis & $\begin{array}{l}\text { Lives alone in a house, no home } \\
\text { office }\end{array}$ \\
\hline P06 & 22 & $\mathrm{~F}$ & $\mathrm{BSC}$ & $\begin{array}{l}\text { Psychology/ } \\
\text { social work }\end{array}$ & None & $\mathrm{N} / \mathrm{A}$ & $\begin{array}{l}\text { Shares room with partner at a flat } \\
\text { share, no home office }\end{array}$ \\
\hline P07 & 40 & $\mathrm{~F}$ & $\mathrm{PhD}$ & Researcher & 1-2 years occasionally & N/A & $\begin{array}{l}\text { Lives at a flat with family, works at } \\
\text { bedroom }\end{array}$ \\
\hline P08 & 30 & $\mathrm{~F}$ & $\mathrm{PhD}$ & Architect & None & Pregnancy & $\begin{array}{l}\text { Lives at a house with family, works } \\
\text { at living room }\end{array}$ \\
\hline P09 & 28 & $\mathrm{~F}$ & $\mathrm{MSc}$ & Researcher & None & $\mathrm{N} / \mathrm{A}$ & $\begin{array}{l}\text { Lives at a house with family, works } \\
\text { at bedroom }\end{array}$ \\
\hline P10 & 40 & $M$ & $\mathrm{PhD}$ & $\begin{array}{l}\text { Medical } \\
\text { Economist }\end{array}$ & 2-3 years occasionally & N/A & $\begin{array}{l}\text { Lives at a flat alone, works at living } \\
\text { room }\end{array}$ \\
\hline P11 & 35 & M & MSc & Researcher & None & $\mathrm{N} / \mathrm{A}$ & $\begin{array}{l}\text { Lives at a house with family, home } \\
\text { office is at garden house }\end{array}$ \\
\hline P12 & 40 & $M$ & MSc & Researcher & 2 years occasionally & $\mathrm{N} / \mathrm{A}$ & $\begin{array}{l}\text { Lives at a house with family, home } \\
\text { office is shared with partner }\end{array}$ \\
\hline $\mathrm{P} 13$ & 37 & M & MSc & Researcher & None & $\begin{array}{l}\text { Attention deficit } \\
\text { hyperactivity disorder }\end{array}$ & $\begin{array}{l}\text { Lives at a flat with partner, home } \\
\text { office in separate room }\end{array}$ \\
\hline
\end{tabular}

pages, Firebase database). Both technologies were used to collect multivariate time series data - through sensors in wearables, and custom sliders in the app-and images and text entries by prompting participants to briefly respond to some questions about their experiences while working.

\section{Wearable Band Mi 2}

Commodity bands Mi2 (Figure 2A) were mainly selected due to their usability advantage and their widespread acceptability and adoption as activity sensing devices. They are easy to use by any individual without any need of technical knowledge or extensive support, and they provide feedback to their users through a display, with vibration and sound notifications. They are already widely adopted in the workplace which endorses the reasoning for selection. The reasoning behind the use of wearable bands for data collection was to explore the potentials of wearables as sensory devices and feedback mechanisms within the context of ambient workspaces. Collected data included step count, distance, heart rate; providing us with a grounding for understanding daily activity while working in the domestic set up. The accuracy of activity data-i.e., distance and step counting - is high as the devices have good accelerometer and gyroscope. Heart rate data were sporadically after user's initiated the measurement. The downsides of commodity bands to provide continuous accurate measurements and pure raw data for analysis were less important for the purpose of this study; whereas their potential to provide feedback as a wearable display were interesting and relevant. Future work will consider higher precision sensors to record activity, heart rate and ambient environment data.

\section{Purpose Built Static Web App: The Affective Workplace Diary}

The static web app ${ }^{2}$ offered an easy-to-use interface for documenting aspects of mood and to characterize the individual workplaces using sliders, text input fields (diary entries) and image uploads. Designed to work as a diary and a survey, it addressed both qualitative and quantitative aspects of the participants daily experiences and mood. The custom sliders $^{3}$ (Figure 2B) were developed for the purpose of selfevaluating mood and the surrounding environment. All sliders ranged, presenting with a default setting of 50 . The sliders that used to evaluate the surrounding environment addressed temperature (cold - hot), light (dark-light), noise (noisyquiet), air quality (poor-fresh). The sliders used to evaluate mood addressed dimensions of mood based on the Circumplex Model of Affect $^{4}$ (Russell et al., 1980; Schimmack and Grob,

${ }^{2}$ The app was developed in HTML5/CSS3/JS, hosted in Github Pages and using Firebase real-time database to timestamp and store the data.

${ }^{3}$ Sliders have a scale of $0-100$ starting from a neutral position (50\%). Any movement either directions was recorded a shift from the neutral position with a percentage and a timestamp. The sliders were changing color from green to light blue without indicating any connotation between color and positive/negative evaluation.

${ }^{4}$ The Circumplex model of Affect (Russell 1980; Schimmack and Grob 2000) served as a basis for developing the sliders around the felt self. Schimmack and Grob model addresses the dimensions of valence (pleasant-unpleasant or positive-negative), arousal (activation-deactivation or energetic-non energetic) and stance (low tensions-high tension). These dimensions are used to map the spectrum of participants mood. 
2000) (Figure 2C). These dimensions are valence (negative-positive), arousal (tired-energetic) and stance (relaxed-tensed). Additional to mood, stress (calm-anxious) motivation (motivated-non motivated) and productivity (productive-non-productive) were included as sliders. The use of colloquial language to address the measures was used to facilitate better communication with the participants. Based on interviews, it did not impact their understanding of the questions addressed. The environment and affect measurement sliders were designed with a reference to validated tools such as the Affective slider (Betella and Verschure, 2016) and were tested with a group of five users prior to the study.

\section{Data Analysis}

Wearable data (distance, step count, heart rate) were exported from the Mi bands by the participants and emailed to the researchers. Web data (pictures, text, slider entries) were exported from the database and organized by the researchers. Interviews were recorded and transcribed using Zoom. Collected datasets were categorized in qualitative data-e.g., pictures, diary entries and interview transcripts-and quantitative data-e.g., slider entries, wearable data-and were further processed and analyzed independently.

\section{Qualitative Data Analysis}

Diary entries and interview transcripts were themed following a thematic analysis approach (Nowell et al., 2017). We coded these transcripts extracting statements on mood and fatigue-e.g., I feel low today, I feel positive today, I did not sleep well, I feel constantly worried etc.,-statements on the experiences and engagement with the physical workspace-e.g., there is too much noise, I changed room I work today, I cleared the notes on my tables, I have a water bottle on my desk etc.,-activities and routines-e.g., I did yoga in the morning, I do small breaks to cook etc., - and finally, on technologies and feedback-e.g., I like the vibration from the band, the web diary made me consider talking to my manager about my anxiety. Pictures (with or without annotations) were qualitatively analyzed and used to support the statements on the experience and engagement with the physical workspace (spatial and ambient aspects).

Qualitative data (text and images) include:

- Interview transcripts of 13 participants-30 min each.

- Daily diary entries of 13 participants for 4 weeks.

- Pictures of spaces (with or without annotations)-number of images per participant varies.

\section{Quantitative Data Analysis}

Multivariant time-series data included repeated measurements of wearable sensory data (distance, step count, heart rate) and self-reported mood and perceived environment data (slider entries, percentage values). Data sets were combined into a dataset and cleaned by the researchers, addressing default and missing values.

Default values were sometimes reported when the users did not manipulate a slider; either because they felt covered by the default value $(50 \%)$, or because they did not have an opinion on the matter at that moment. Additionally, heart rate data were often sparse. We chose to approach these entries as missing data (N/A). Missing entries were addressed through linear
A
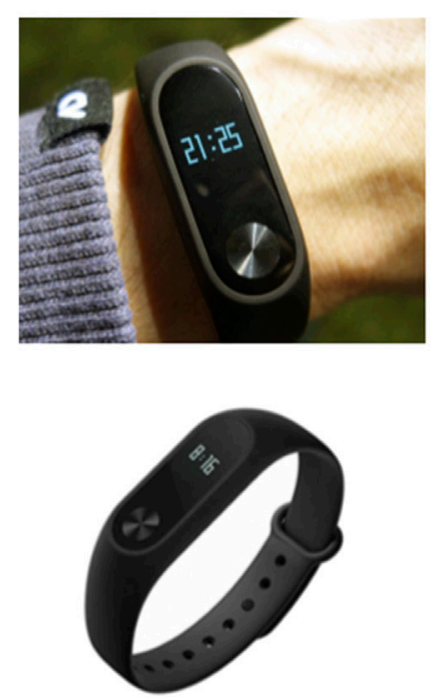

B

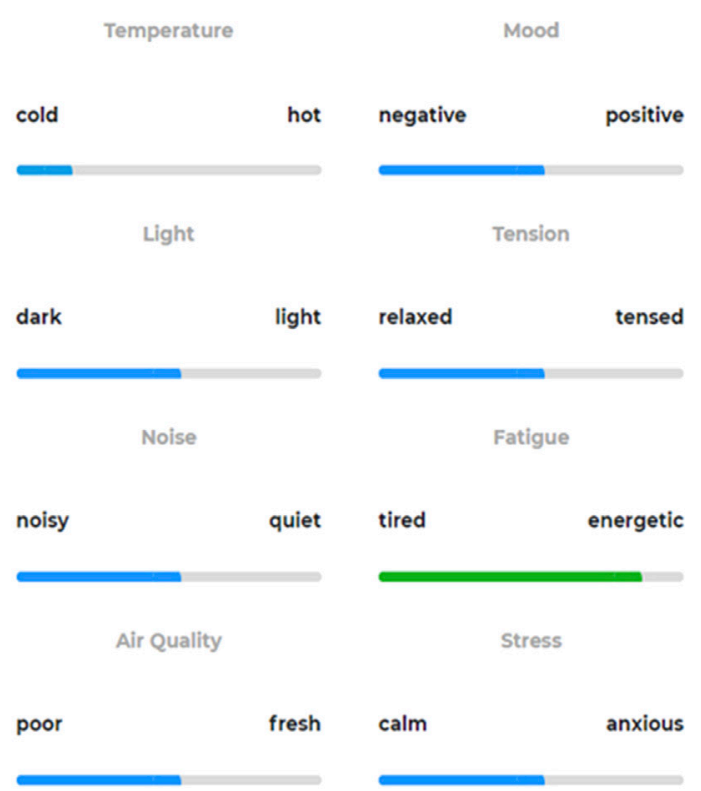

C

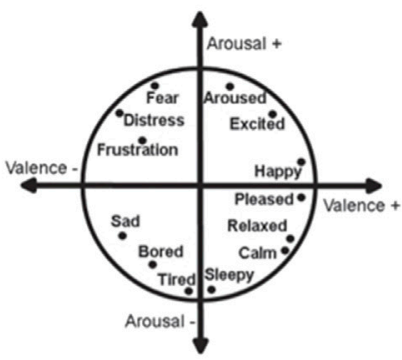

(a) Russell's Circumplex Model



(b) Schimmack and Grob Model

FIGURE 2 | A Mi Band 2 wearable used for the study, B Purpose-build sliders for self-reporting, C dimensions of affect models. 
interpolation using the ImputeTS library and the $\mathrm{kNN}$ (k-nearest neighbor) in the sum of participants data. We consider that N/A values had limited impact on our findings.

Datasets were then processed in $\mathrm{R}$ studio following relevant methods (see rmcorr, auto-sklearn) to use the most of their statistical power. The analysis followed two main pathways, 1) exploring multivariant correlations and 2) testing the predictive power of the data:

1) Correlation analysis of multivariant time-series data: selfreported mood-e.g., valence, arousal, stance dimensions, stress and motivation, perceived aspects of the environment-e.g., light, noise, temperature, air quality entries-and wearable data (steps, heart rate). The aim of this analysis was to unveil multivariant correlation patterns in the sum of participants data. Correlation methods were first applied the sum of data using corrplot to establish correlation coefficients; then in pairs of variables within and between participants using rmcorr (Jonathan and Laura, 2017) to establish the statistical significance of results.

2) Prediction of dimensions of mood based on self-reported ambient environment and wearable data. This analysis explores the potential of predicting mood solely from variables that can be captured using passive sensing, to draft the design implications and the feasibility of automated predictions for future work. ML models applied were decision trees and random forests to establish the predictive power of various variables; and multiple automated ensemble learners using auto-sklearn (python) (Feurer et al., 2020) to explore the potentials of predicting valence based on environmental data only.

\section{RESULTS}

\section{Qualitative Data Findings}

Through the qualitative analysis we explored three themes, which are unpacked further below: making a space for work at home; the challenges of embodied wellbeing in the lived workspace; and the value of feedback for the remote home worker.

\section{Making a Space for Work at Home}

Bringing the "office" home requires specific space use modifications-such as finding a space or a corner, having a desk (or a table), a chair, one or more screens etc.- to take place. Participants tried to satisfy these criteria following different approaches. As a result, domestic lived workspaces look necessarily messy and chaotic. Analyzing participants' pictures, we identified some underlying principles that they follow to structure their home offices. These principles could be described as a) supporting basic ergonomics-e.g., surface leveling, positioning and orientation of displays b) forming visual cues and peripheral constraints-e.g., visual barriers to limit external distractions and guide focus, c) supporting comfort-e.g., across multiple dimensions such as kinesthetic, visual, thermal, auditory, etc., and d) providing positive engagement-e.g., objects for relief and self-entertainment. Due to different needs and the lack of space, diverse approaches to structuring spaces through the above principles are discussed below, drawing on the pictures and diary quotes participants provided.

Basic Ergonomics in Limited Space: Leveling and Displays During interviews, 7/13 participants mentioned that they prefer having a fixed home-office set up and have the option to alternate between working at home or at the office. 5/13 participants stated that they need their home office to have a good chair and a table/desk surface that can fit a monitor, their lap-tops and notebooks as minimum requirements. Congestion of objects due to lack of space was a common theme as seen in their pictures (see Figures $\mathbf{3 A}, \mathbf{B}-\mathrm{P} 07)$, resulting in poor ergonomic arrangement besides their best efforts.

Most of the connected workers spend their days in front of a screen and many mentioned their need for more and bigger display areas. A few brought extra monitors at home $(6 / 13)$.

"We have we had one separate screen at the office; having more screen space helped me a lot. I sort of wanted to replicate that a bit when working from home. I set things up with two monitors plus my laptop and I find that I find like work better that way." P01

A few (4/13) mentioned that they used a spot with stable screens and their move their lap-top around the house if they need to change spot. Some (6/13) used solutions such as lap top risers or trays to increase their work surface, bring objects in the right reach zone, or position displays in the right visual height. P03 uses a lap top riser utilizing surface space when working with her partner (right) or in the living room (left), while bringing the display to the right eye-level (see Figures 3C,D).

\section{Visual Cues and Peripheral Constraints}

A few participants mentioned (4/13) using their desks or surrounding wall surfaces as display extensions, sticking notes as reminders. Three (3) participants mentioned piling post-it on their desk relevant to online meetings or the tasks they are working on that week and changing them when the task changes.

"I clean all my notes on the desk when I start on a new project” $\mathrm{P} 07$.

Observing the pictures, a few (4/13) prefer having a neutral periphery with their only focus point on their screens, whereas others populate their desktop periphery manually extending their display spaces (see Figures 4A,B-P12 below).

Apart from expanding their displays in the periphery, there is also a need to constrain it. A few used covers or furniture 

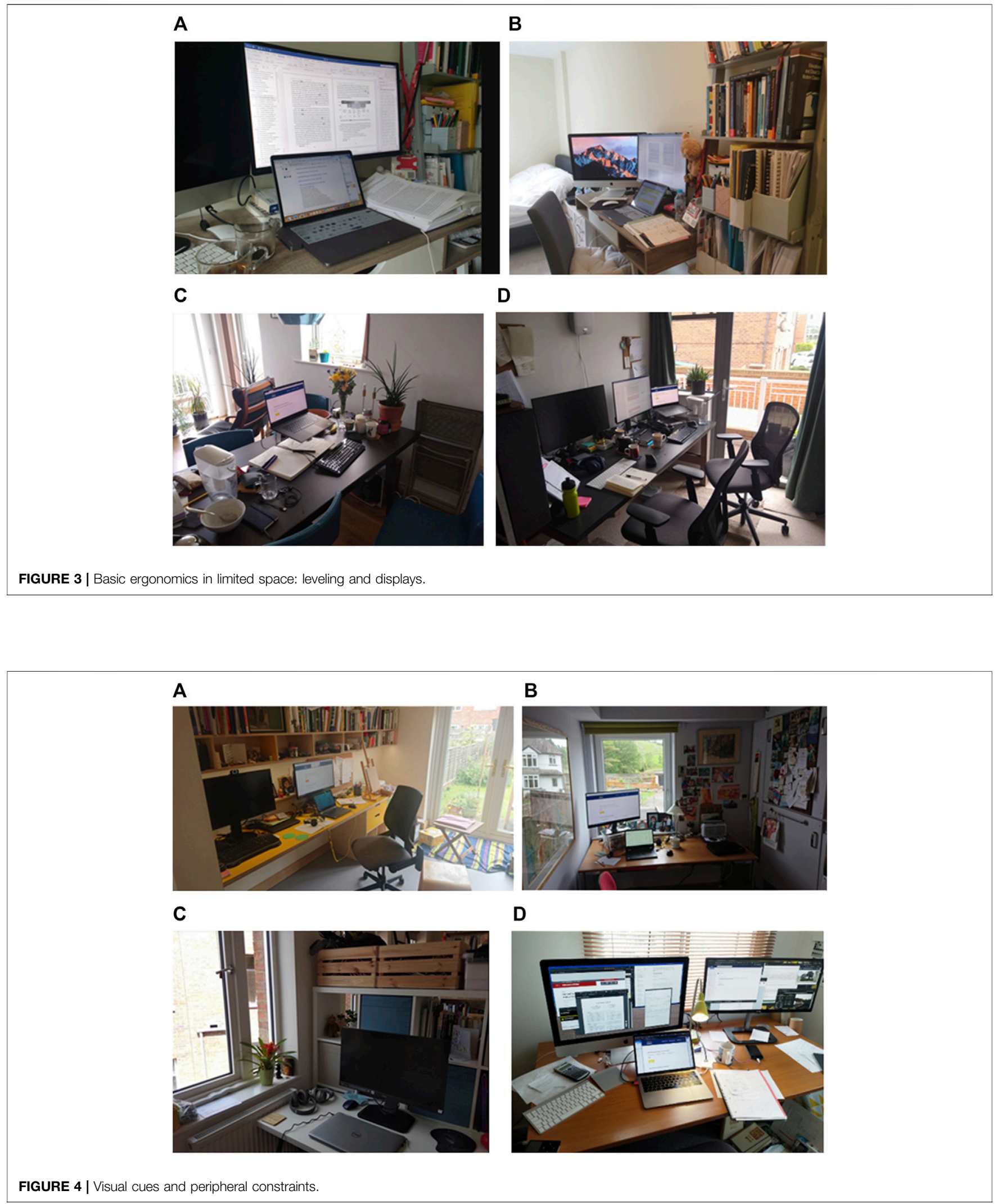
A

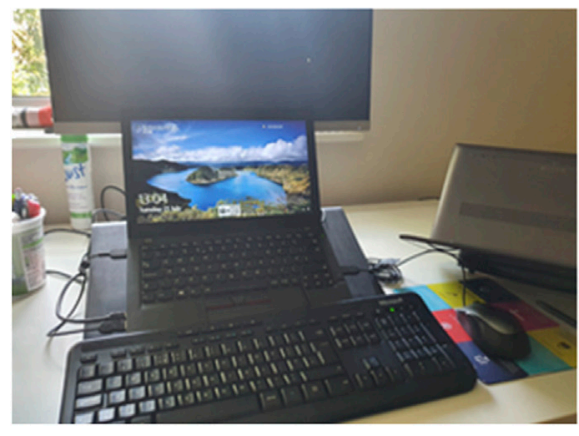

B



FIGURE 5 | Comfort and ambient aspects.

as elements to block or filter visual distractions. In Figure 4C, P13 uses a shelving unit that blocks view in the rest of the room, while P01 (Figure 4D) uses blinds to manage light and the view.

\section{Comfort and Ambient Aspects}

Comfort has many dimensions, such as visual, auditory, thermal, and kinesthetic. Often these are hard to distinguish as discrete elements in the experiences of the participants. The discussion bellow highlights participants' actions to maximize their comfort, addressing aspects of natural light, visual contact with the outside, ambient color, and noise.

Ambient natural light came in many quotes as essential. Observing the participant's work desks, most of them face a wall with a window -the source of natural light- located either on the right or left side; with only three of them facing it. Most of them prefer natural light coming on the right or left side of their desks, avoiding getting blinded. A few (2/13) repositioned, rotated, or levelled their displays to balance blurring and the direction of light coming in the room. Some (4/13) mentioned that they avoid facing their windows while their work - direct visual contact with the outside - to avoid visual distractions such as movements of cars or people. The few that choose to face their windows mentioned enjoying ambient movement and visual contact with nature while they work $(2 / 13)$.

"I like the movement from the construction site outside the window, it feels like something is going on. [...] I used to work on the train, I liked the movement outside the windows." P01

In Figures 5A,B below changing their screen positioning depending on the ambient light in the room (P10) while maximizing visual contact with nature-using the window as a calming display. It was also common in participant diaries to refer to the weather and the colour of the sky, in relation with their mood (12/13): "I feel a bit low today, and it is so grey and rainy." P02 A few mentioned using white sound (2/13) and nature soundscapes (1/13) to neutralize or cover noise from outside. Many mentioned that they enjoy listening to music without headphones in their home office (4/13).

Some mentioned using day light lamps to balance insufficient natural light in the room or extend their working hours $(2 / 13)$, positioning day-light lamps on their right and left sides of their work desks. Diary quotes and images suggest that $7 / 13$ generally prefer neutral-coloured spaces to work, with a few (4/13) prefer more colourful spaces, adding posters in their periphery.

"I need a neutral space to work, I try to neutralize the environment around me from sounds or colors, and make sure that it is not something I update every day." P07

Some participants (2/13) said that they found different spaces in their home to offer different dynamics - "more creative tasks can happen in more colorful and messy spaces" P13, whereas "thinking requires neutral spaces" P07 (Figures 6A,B). A few said (3/13) they choose their spots depending on what they are working on, how they feel or depending on the weather and light in the house.

I think if I need to be bit creative it's better for me to sit in the living room where there are more colors ... more colors can also work distracting. If I need to concentrate, I find concentrating there harder.” P13

\section{Positive Engagement with the Workplace}

Finally, plants, decorative elements, de-stress balls, music instruments were very commonly placed in the participants periphery to enhance positive engagement with their workplace (Figures 6C,D).

\section{The Challenges of Embodied Wellbeing in the Lived Domestic Workspace}

Changing spots: conflicting need for variation, structure, and flexibility. Several participants (4/13) mentioned changing workspaces as an inherent part of their workday when working outside home, visiting different spaces in a building, or working in different buildings. Many mentioned missing the variation of spatial experiences when working from home, and that it is something hard to re-create.

"I used to work from the library or from the office and then go and teach in the medical sciences faculty. Then, suddenly, it is just one room." P09 

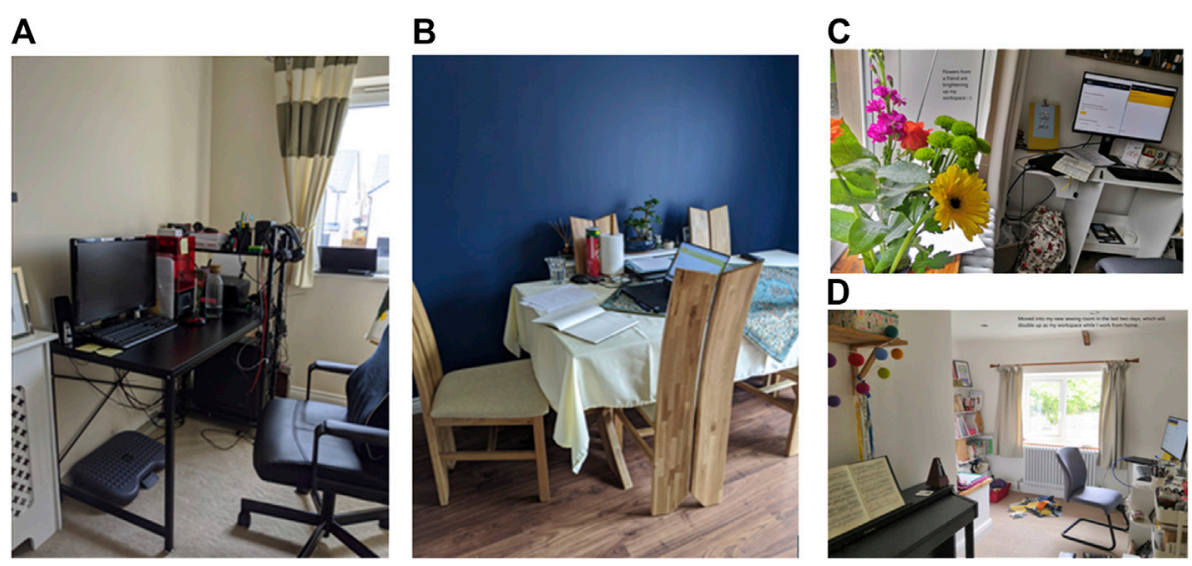

FIGURE 6 | Positive engagement with the workplace.

Still, the domestic workspace often requires a level of flexibility to balance conflicting needs. Some said $(6 / 13)$ that they need to have a fixed workspace in their houses, while others (5/13) claimed to be more flexible and work in their living areas, kitchen tables or often change locations in the house. Three more participants mentioned that besides their preferred fixed spots, they sometimes alternate locations in the house to satisfy family life.

"I really do not mind working on the sofa or the kitchen bench; Wi-Fi is actually better here." P05

"I work in whichever room is available. Currently I have a desk in our sewing room, next week I will work at the dining table." P04

The more "flexible" participants $(5 / 13)$ mentioned that they can "blend" their workplace with other domestic spaces without having critical focus issues or task-prioritization conflicts. A few $(2 / 13)$ mentioned that changing work spots help them regain focus.

"I realized I like changing space for working, it helps with focus attention.” P02

On the other hand, the participants (6/13) that seemed to be more sensitive on their set up mentioned that they prefer to have a separate workspace from the living areas, keeping a clear division between "work" and "domestic" life. A few of them mentioned challenges to do so, such as the lack of space and conflicting priorities between domestic and work life, or different aspects of remote work in the same space.

"We share the office room and desk with my partner as we tried to separate our work from the living space. We do not have much space as you can see in the pictures. If one has an online meeting at the office space, the other moves in the living room so we do not disturb each other." P03

\section{The Lack of Rhythm and Pacing}

Many mentioned the lack of "rhythm" during their day, often occurred from informal chats with colleagues. With no informal social interactions at place, it seems that both the engagement with work and the quality of breaks was getting poorer (3/13).

\section{"The working day needs a rhythm. working from home is constantly relaxing or static. There is no variation or change." P11 \\ "Now actually need to sort of entertain yourself to actually have breaks. you're sort of beating yourself up about not maybe doing enough work and then on the other hand, you're not taking off like quality, quality breaks, either" $\mathrm{P} 03$}

A few mentioned that they feel that the lack of pace in their workday results to them feeling too relaxed, unproductive, and extend their working hours (2/13). Similarly, many participants find hard to zone in or out of work in their homes, unless they leave their rooms. A few find alternating between domestic and work life easier (3/13).

"Being too flexible results being unstructured or not having a clear routine $[\ldots]$ my schedule becomes too irregular, and I work until very late at night." P11

"I do not have an issue with zoning out of work in the living room, I just turn my lap top off." P05

\section{The Lack of Movement}

Physical activity is important for health daily work life. 10/13 of the participants reconned the importance of daily movement as part of their workday. As connected workers do most of the work in front of a screen, many mentioned (9/13) that they walk much less at home, as there is no need to go somewhere and the distances in the house are much smaller.

"At the office, I would walk to meetings, rather than do $3 \mathrm{~h}$ of video meetings. Walking would be part of my 
routine, because normally I would walk across the campus to another meeting room. I don't really walk much when I work at my house. I go up maybe for a cup of tea, you know, that sort of thing." P04

"When I go to the office, I have to run around between the warehouse and my desk. That is why I walk more. Whereas when I am at home, I am just with myself and the computer." P08

A few mentioned that they "forget" to move away from their chairs for long time as they work uninterrupted. A few reported increased fatigue (3/13) and eye conditions from long screen staring (1/13). Many reported lower backpain (6/13) from sitting too long at their work desks. Back pain problems also rise due to the use of nonergonomic furniture -such as kitchen chairs- as office furniture, resulting sitting long in the wrong postures. A few (2/13) also referred to irregular breathing-e.g., holding their breath due to stress or concentration-relating it with fatigue their experiencing.

"I have back and neck pain again, I have been sitting too long to concentrate on research [...] I feel I am not productive enough, and no-one interrupts me. You tend to forget about the time, you forget to do breaks." P11

"Because I am concentrating on a project, sometimes I think stopped breathing properly. I believe that is kind of thing, is making me fatigue because of the lack of oxygen." P11

Some use different furniture to work (3/13) and avoid sitting too long in the same posture; or take short breaks to lay back.

"I get a lot of lower back pain because of sitting too long. One good thing of being at home is that I can take short breaks to lay back on the sofa and relax." P08

Finally, a few observed how physical activity helps them thing and changes their experience of the surroundings while working. They mentioned that physical activity helps them deal with noise distractions; feeling better because of "fresh air," and that lack of physical activity changes their perception of temperature.

"Walking works for me, it gives me a lot of time to think. When I'm coming back, I am more efficient. It is not that noise becomes less prominent, but you are more resilient to accept the distractions than before." P07

"I start feeling cold if I sit too long; I know it is not colder than usual, but it feels like it is." P13

Most participants refer to restoring from bad mood and fatigue is through outdoor and indoor physical activity, either pre-planned or spontaneous. Many mentioned taking short active breaks during workday- stand up to stretch, yoga, walk or jump inside the house (5/13), plan a physical activity to start or end the workday (4/13) as "waking up ritual" and "a mental preparation for work," and practicing breathing techniques to relax. (1/13).
"I just jumped around the house to shake off the tension of writing the paper. [...] During lunch I did some yoga, which was nice to recharge for the rest of the day." P03

"As every day, I started the day with yoga \& I practiced Buddhism (morning/evening).” P02

"What really helps to control my stress is focusing on my breath, doing a breathing meditation-I practice box breathing for a few minutes while I work." P08

\section{Distractions in Isolation}

Noise from construction sites or the street was mentioned most in the diaries and interviews as one of the main causes causing cognitive overload, irritation and fatigue while working (4/13). Escaping from noise while working at home is a challenge; some mentioned shifting their working -hours resulting in working late or closing windows-resulting in low air quality and high temperature.

"For example, I close the windows because there's a building site next to our house and they can be really noisy throughout the day. But it's very irregular.” P13

Other mentioned that distractions "are often more internal than external. It is a lot about managing own's thoughts and emotions" P13, referring to persistent negative thoughts and emotions (4/13). A few mentioned getting "stuck" in a negative mindset regarding their work or themselves without being able to "get out" (4/13). Some addressed (2/13) that it is hard to stick to what is good for you; being unable to manage yourself in a way that is good for you or do anything to improve the way you feel.
"If you don't feel so well, I think you don't have the discipline to do the things that are good for you. Because they often need some engagement, you need to make it choice to stop and start again. And to be able to do that, you need some mental energy." P13
"If something goes wrong, I have no one to bounce off at all. If I get into a negative like or stress kind of headspace, I just stay in it.” P04

Finally, some mentioned being overloaded and distracted by notifications from diverse collaboration tools and social media for work $(3 / 13)$.

"We have three different platforms that we communicate with my colleagues. One day last week all three of them were buzzing non-stop. It was email, then teams, and discord which we use for community. I keep this window open and try to answer all of them, but it was a bit crazy to deal with all of these. If you are in our office, you just talk to the person." P08 


\section{The Value of Feedback for the Remote Worker}

Many participants mentioned that since working alone, they relied even more on following a schedule and setting notifications to make sure they keep up with it (8/13). A few of them used tools to help them structure their workday and support their wellbeing $(3 / 13)$; alarms to manage their schedule (2/13), reminders for activity breaks (2/13), while some went further checking their breathing patterns (2/ 13) or controlling calories (2/13):

"I use pomodoro to set up working intervals and make sure I take regular breaks.” P03

"I bought a band to check the oxygen saturation levels in my blood; I believe that I get fatigued because of the lack of oxygen.” P11

Below the participants opinions around the use of the provided wearable devices and the mood-measuring tools in the context of domestic workplace.

\section{Feedback to Move}

Many participants (10/13) found the band pleasant to wear and non- intrusive. A few said that their daily exercise was somehow fixed and did not need any reminders for this (5/13) or that the band was not accurate in terms of measuring other types of activity such as yoga or cycling (4/13).

"I do a walk or a cycle in the morning anyway; it is irrelevant with the band [...] I am not a smart person motivated by targets anyway." P01

"I do yoga every morning, but I feel it does not measure that ... it only measures steps." P02

Still, many mentioned that it made them more aware of the fact that they walk less during their workday through the display of steps (6/13), and that the band worked very well for this purpose. Some stated that it was more effective than mobile apps, as you wear it all the time. Some mentioned that they use it to set up a daily step goal (3/13) and tried to keep up with it.

"I didn't set any particular targets, but when you check the steps and see how little you have walked ... well it motivates you to move." P06

"I have a mobile app for step counting and calories, but it is not as effective as the band-it is always on your hand." P10

Others used the alarm to set up regular breaks from work (3/ 13). They mentioned that the vibration was pleasant as a movement reminder, whereas noise or voice interruptions where not that positive.

"I loved waking up with the vibration, it was a nice experience." P02

"... also the band vibrates and reminds me to take often breaks." P04

\section{Feedback for Self-Reflection}

Many participants (5/13) mentioned that it was hard to describe and evaluate how they actually feel during their workday. As they get absorbed in their work in remote, they mentioned not paying much attention to themselves (e.g., their body-posture) their surroundings (e.g., working under poor lighting), or their mood while working (e.g., getting caught in a bad mood). The ways they described their mood in their diaries can have many interpretations; they often made associations with to explain how they feel to themselves-i.e., I feel low because I had bad sleep, the weather is bad, I have too much work, or there is too much noise and that irritates me etc.,-but it is unclear if these aspects are causing the problem or they come to their attention because they feel low. Using the sliders was meaningful in that case to provide a mental anchor and a measure that they can assign to how they feel. Many mentioned that the diary and the sliders initiated their emotional self-reflection by setting a scale and aspects to consider; others claimed that it helped them in trying to understand potential causes of it. A few (3/13) mentioned that they started checking their environment and work set up more often to make sure they have adequate light etc.

"I actually start observing how the light and air quality is while I am working at how it might affect me.” P13

A few noted that they enjoyed kept diaries trying to see patterns in their emotions and thinking (4/13) whereas others preferred manipulating the sliders over writing extensive text (4/13).

"I found the diary meaningful to try to understand my thought/emotions patterns better and find ways to manage it." P07

A few mentioned that the self-reflection exercise was meaningful for self-management (3/13); and that it pushed them to take action on changing what caused them discomfort (2/13).

"Participating was an interesting self-reflection exercise for me. The study pushed me to realize I was not alright and talk to my manager about the fact that I am constantly anxious.” P06

Others mentioned that being made aware of their emotions and learning to accept them was an interesting part of the study (5/13). The majority found the diary study unobtrusive, but a bit tiring and repetitive, suggesting that more variation in the questions based on context and previous entries would be meaningful. Some pointed to further considerations and improvements, such as:

"Allowing better self-management through visualizing mood and activity circles." P11

"I would like to be able to see a visualization of my mood fluctuations." P02

"I would like to have an archive of my previous entries to understand my mood and thought patterns." P07 
"I would like to have more personalized questions based on previous answers I gave, to help me gain deeper insights to what actions can help me." P12

From Awareness to Changing Behavior: Reflecting on Diverse Attitudes on Feedback

In summary, there were diverse reported attitudes regarding the impact of the wearable and diary feedback. A few (4/13) responded that feedback was a merely self - confirmation:

"I was feeling tired, the survey made it clear to me that I need a holiday" P04

"I know I do not walk much, the band just confirmed it." P04

The majority mentioned that the web diary increased their "self-awareness" about their own mood fluctuations and thought patterns $(6 / 13)$ and helped them cope with the situation $(2 / 13)$ or nudged them to act $(2 / 13)$. Participants mentioned that they used the bands to set reminders and goals to move more often; and that they were useful in pushing them to have regular breaks. Still, it is unclear if the band helped participants to form healthier habits in the long run and without "on the spot" reminders. 7/13 stated they will keep using the band after the study; but we do not have data on how they use it and if their behavior shifted.

\section{Quantitative Data Findings}

Aspects of the Environment and Embodied Wellbeing: A Multivariant Correlation Analysis

This part of the analysis focuses on framing correlations between repeated measurements of self-reported mood and ambient environment, and activity and heart rate data. Multivariant timeseries data included wearable activity data (distance, step count, heart rate), and self-reported data on mood (valence, arousal, stance, stress) and perceived environment (light, air quality, temperature, noise) recording values from 1 to 100 from the custom sliders.

Figure 7 illustrates the correlation coefficients for all mood and environment correlation combinations. The analysis shows a moderate positive correlation $(R=.54)$ between perceived air quality (poor-fresh air) and valence (negative-positive mood). The analysis also shows a mild negative correlation between air quality and stance $(R=-.36)$ - i.e., decrease of air-quality and increase of tension- and stress $(R=-.22)$-i.e., decrease of airquality and increase of stress. There is a very mild positive correlation $(R=.23)$ between arousal and air quality-i.e., feeling energetic when perceived air quality increases. The analysis also showed minor positive correlations between noise and valence $(R=.25)$-i.e., positive mood in quiet workspaces - and light and valence $(R=.19)$-i.e., positive mood in more well-lit spaces. While these correlations do not represent a particularly strong association, noise and light were frequently mentioned in the qualitative data.

Similar results occur using rmcorr - repeated measures correlation - highlighting the impact of air-quality on mood, also establishing the statistical significance of these findings. Below (see Figure 8) the correlation graphs of the most significant $R$ and $p$-values for pairs of variables in the sum of participants; each participant is represented in a different color.

We also examined the relationships between self-reported mood dimensions to understand how these relate with one another and validate data coherency. Valence and arousal have a mild positive correlation with no statistical significance level $(R=.37, p=2.44)$ meaning that feeling energetic loosely correlates
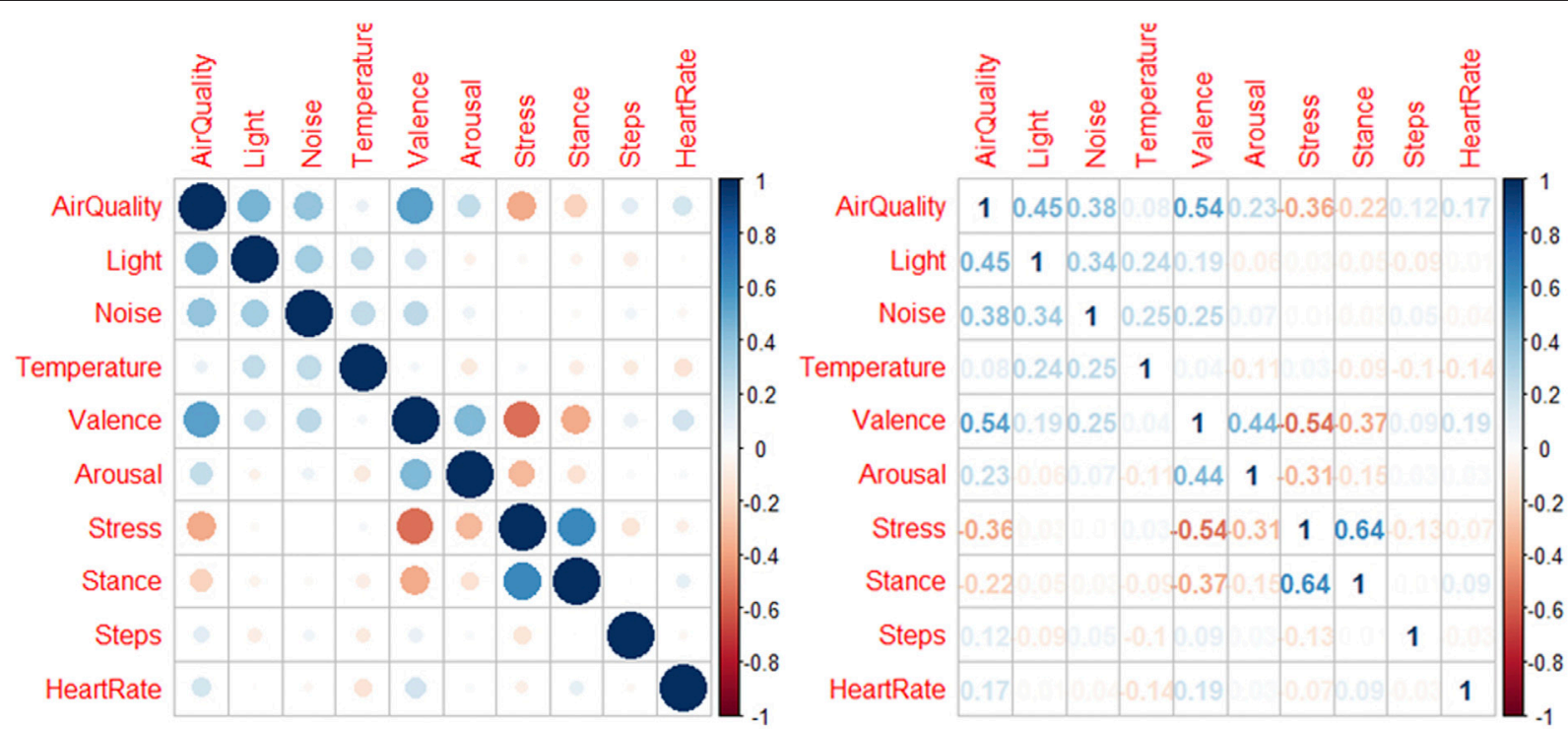

FIGURE 7 | Correlation coefficients for mood and environmental variables. 


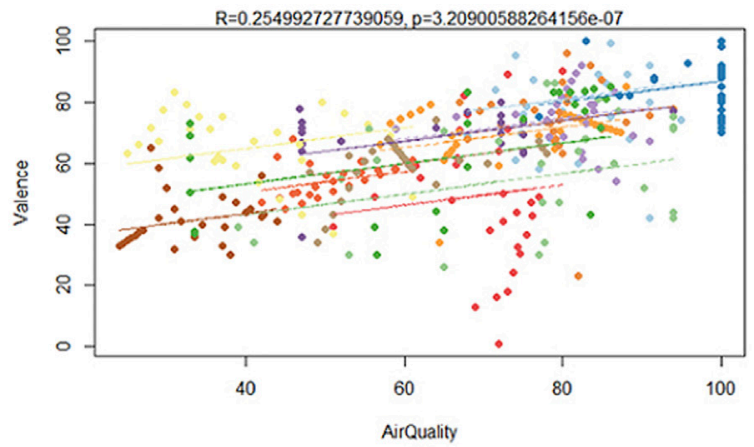

Valence- Air-quality: Moderate positive correlation not statistically significant $(R=0.25, p=3.2)$

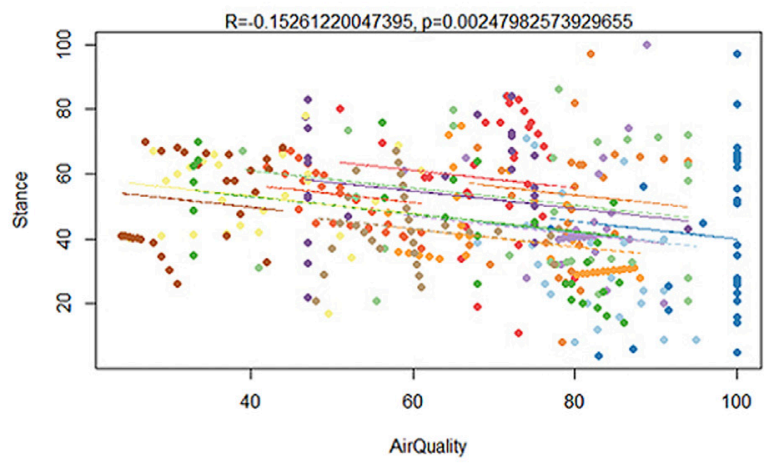

Stance- Air-quality: Mild negative correlation, statistically significant $(\mathrm{R}=-0.15, \mathrm{p}=0.002)$

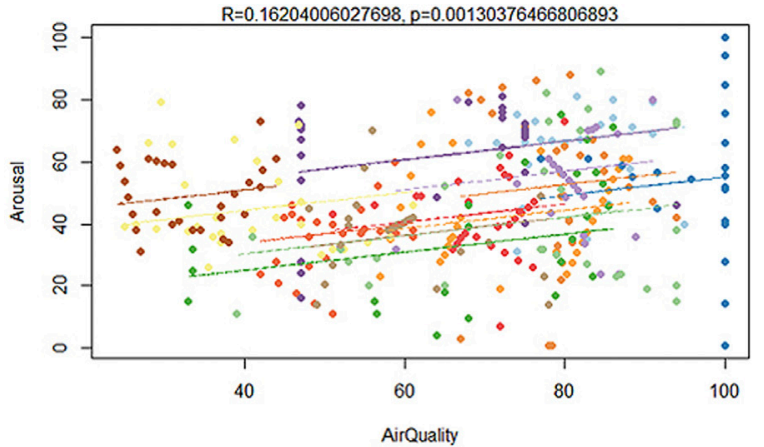

Arousal - Air-quality: Mild positive correlation, statistically significant $(R=0.16, p=0.001)$

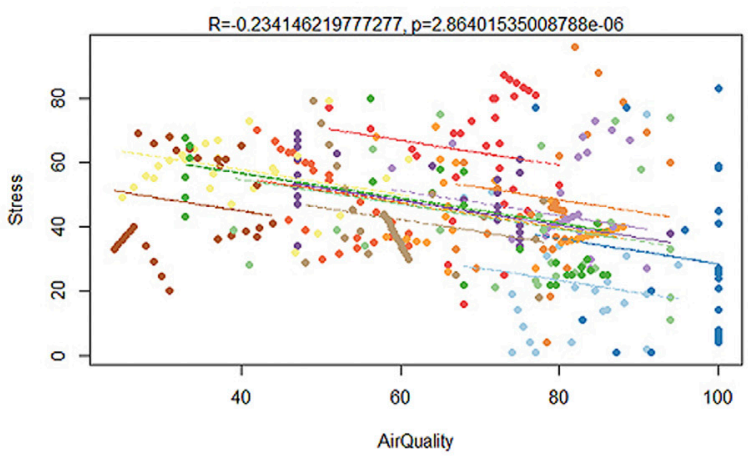

Stress- Air-quality: Mild negative correlation, not statistically significant $(R=-0.15, p=2.86)$ with feeling positive; similarly feeling in low mood loosely correlates with higher tension.

The outcomes indicate a relationship between air quality and valence motivating further study, as passive sensing devices for air quality are readily available; yet the persistence of the association with passively collected sensing data for the context discussed in this work remains to be validated with a greater sample of participants and sensory measurements.

\section{Predictive Potential for Mood Based on Passive Environmental Sensing}

This section further explores the possibility of predicting selfreported affective states from environmental measures. We modelled the prediction of each of the parameters: valence, arousal and stance; based on the self-reported environmental data (light, temperature, noise, air quality) and the passively collected activity data (steps, heart rate). Random forest training and multiple regression modelling approaches suggest that environmental data have a greater predictive power on valence than the rest of self-reported mood data (stance or arousal). We continued with exploring predictive potentials of valence using decision tree modelling to obtain an explainable model based on solely environmental data. This selection was based on the results of multiple regression modelling and a categorical separation of environmental and activity data. The decision tree produces the diagram below (see Figure 9), highlighting the importance of air quality (see top node and layer 2). The confidence level is above $90 \%$ for each node. Addressing the extremes, Node 10 suggests that if Air Quality obtains a value above 77.75 (i.e., fresh air) and Temperature a value below 47.25 (i.e., cool temperature) Valence would have a value of 85 -i.e., very positive valence when working in fresh air and cool temperature. Node 4 indicates a low valence (below 40) occurs when noise is high (below 41) and air quality is low (below 56). This approach provides the basis of setting rules and constraints in design of future user-driven systems, given that inputs (Air Quality, Light, Noise and Temperature) will be actual sensory measurements.

Finally, automated predictive ensemble composition using the machine learning (ML) package auto-sklearn (Feurer et al., 2020) was used to explore best candidate regression performance to predict valence ratings based on environmental factors. The model indicates a strong correlation (R2: .7) when employing a $240 \mathrm{~s}$ runtime limitation with a threshold of $30 \mathrm{~s}$ per individual model training with a feature set of: air quality, light, noise, temperature, steps, distance, and heart rate. Prediction based on the activity sensing data alone resulted only in a weak association (R2: .3). For practical purposes, using the same framework and runtime settings, we also explored the medium or high values for self-reported Valence, using automated exploration (auto-ML) of 


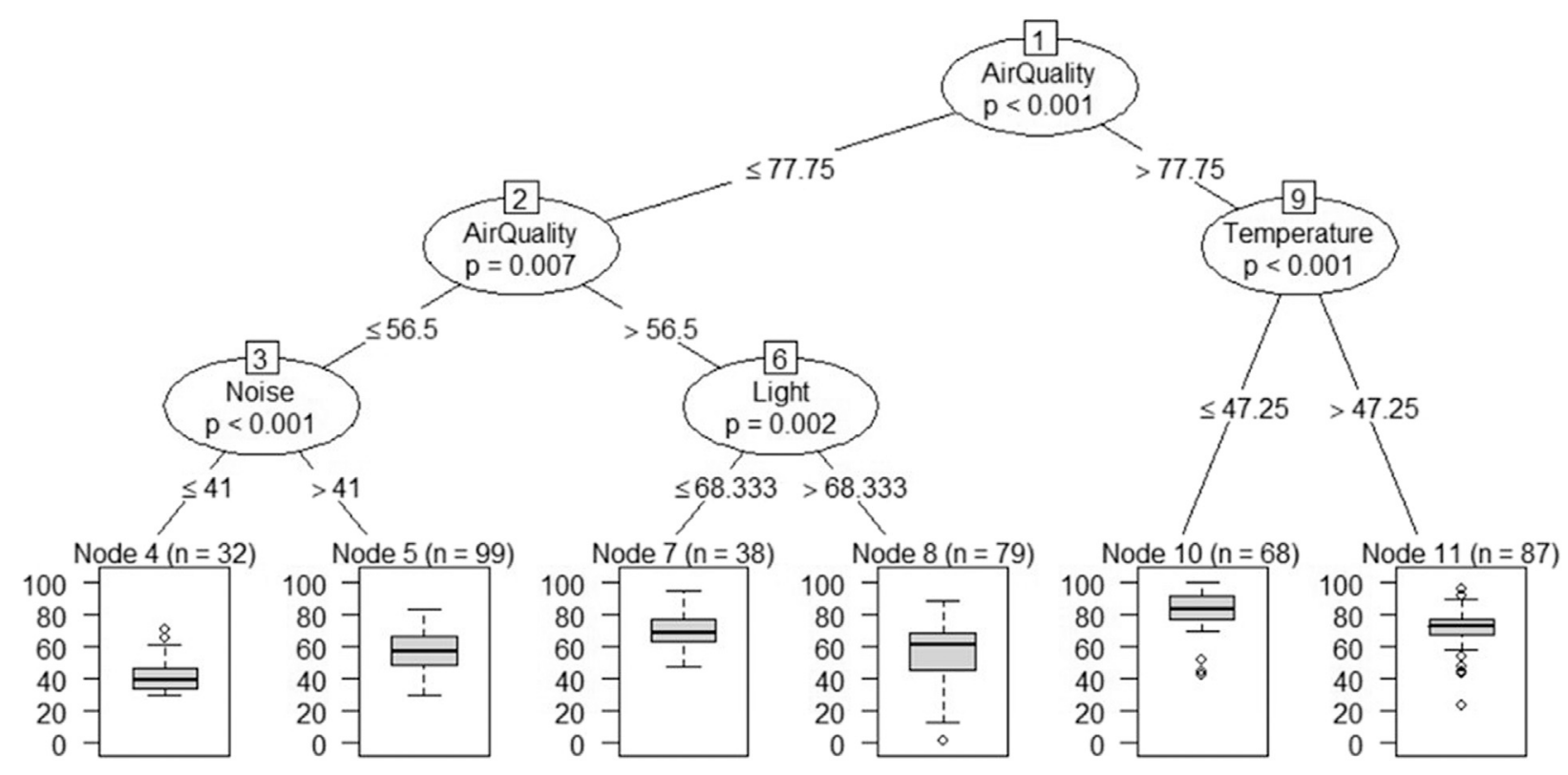

FIGURE 9 | Decision tree diagram: environment and mood data.

a structured hypothesis space of 110 hyperparameters (based on 15 ML models). The resulting ensemble indicated an overall best possible accuracy with the given data set of $80 \%$ (avg. precision .82 , recall .63) after pruning to three features only (air quality, light and noise). Automated ML ensemble generation indicates that predicting mood from passive data may likely be possible, showing $80 \%$ accuracy in predicting low/medium/high valence classes and an R2 of 0.3 for prediction via regression.

We acknowledge that this remains a group-level exploration and early indicative findings for now, that indicate a good reason to aim for further validation using passively collected ambient data and a greater sample of participants and repeated measurements. If that is successful, individualized models should also be explored as they can reasonably be employed for workplace usage and would likely lead to much more reliable predictions - as they would respect individual characteristics and contextual information from the workplace.

\section{DISCUSSION}

\section{Reflecting on Field Observations}

The analysis of collected data has provided some interesting and significant findings in relation with our research questions (RQ1, RQ2, RQ3, RQ4). The qualitative results addressed the physical and embodied challenges of the engagement with the domestic workplace such as the lack of movement and the difficulties transitioning between working and domestic life (RQ1, RQ2). The quantitative data highlighted significant correlation between air quality and mood and highlighted the potentials of predicting mood in remote home offices based on passive data capture (environmental data, activity data) (RQ3).

The challenges of managing office ergonomics in limited, often shared workspaces, and with diverse space-use were highlighted in diary quotes and pictures (RQ1, RQ2). Participants illustrated a diversity in their needs for spatial structure, flexibility and spatial variation while working from home. Participants had different needs in terms of structure to engage with work and expressed different levels of spatial autonomy with regards to their work tasks (RQ1). Some participants took steps to deal with the problems of limited space adapting levelling, positionality and orientation of surfaces-e.g., creating barriers or using stands. Changing spaces and manipulation of spatial affordances was mentioned with regards to positive engagement and zoning out of work (RQ1). These actions point towards designing for work surfaces with a greater adaptability to match with the lack of work areas and the changeable space-use needs, while ensuring ergonomics and comfort (RQ1, RQ2). The work of Petersen and Grønbæk et al. (Takashima et al., 2016; Grønbæk et al., 2017; Grønbæk et al., 2020; Petersen et al., 2020) is an example of such interventions with a focus on informal collaborative work in office spaces; whereby shape changing interactive desks initiate dynamic work areas. Moving forward, bringing adaptive furniture examples to remote home workspaces would address enabling calmer transitions between domestic and work life (Stawarz et al., 2013; Toch et al., 2020), and assist with different aspects of remote connected work (Deloitte LLP, 2018).

The need for multiple display areas and the intuitive expansion of display in the periphery-e.g., the piling and cleaning of notes-point towards extending ambient work displays to the surrounding physical environment (RQ1). Reflecting the work on projected AR displays (Huber, 2014; Winkler et al., 2014; Lee et al., 2019), tangible interaction can extend work tasks beyond the screen. Other aspects mentioned were feeling disconnected with colleagues, missing spatial variation and rhythmicity of the office space, and getting overloaded from using many online collaboration tools that often fail to address their needs (RQ2). 
Moving forward, research on extending the display could help reduce cognitive load from long screen exposure and notifications (Ludden and Meekhof, 2016; Bittner et al., 2019), while engaging and interacting naturally with the physical space; remote collaboration involving spatial interactions and enhancing copresence of colleagues-bringing the rhythmicity and natural flow of the workday to remote settings.

Regarding ambient aspects and wellbeing (RQ3), noise was mentioned as a core issue as well as the lack of natural light. The participants intuitively positioned their work desks and displays to better use ambient natural light in the room, enhanced natural light in the rooms using day-lamps, and used windows as light and calming displays-e.g., providing a view of nature (RQ1). Some mentioned that different ambient light and ambient colour impacts their mood and productivity differently, relating colour to creative work tasks (RQ1). Reflecting past literature (Rogers et al., 2010; Snyder et al., 2015; Pereira et al., 2016; Yu et al., 2018), ambient light has been used as mood feedback, stress biofeedback, eco-feedback and restoring strategy (Yu et al., 2018). Moving forward, ambient light can be explored as a social and selfawareness strategy in remote workplace; as "mood and environment" feedback, and in interaction with voice data.

From a quantitative perspective, air quality showed strong correlations with affect-e.g., positive mood and perceived fresh air quality-, particularly valence (positive- negative mood) and secondarily with arousal (low-high energy) (RQ3). This is a very interesting aspect that appears only indirectly in the diary entries, but it was picked up when directly addressed. This suggests that air quality is an aspect that requires "surfacing" to be brought to the occupants' awareness (RQ3). The interpretation of the correlation of air quality to valence accounts broader considerations relevant with the data collection methods. As these measures are based on self-reported and not sensory data, there are multiple interpretations assigned to air quality such as low oxygen, low air-circulation, high temperature, high humidity, and the combination of these aspects. Poor air quality in terms of the experience of remote workers could also relate to some reported breathing irregularities (RQ3)-e.g., participants breath less due to stress or concentration, and therefore report bad air quality. The negative correlation between stress or stance and air quality also points towards this association.

Nevertheless, the correlation of air quality with valence-e.g., good air quality makes building occupants happier-is interesting (RQ3) and needs to be validated with greater samples and sensory readings in future work; also identifying which aspects of air quality-i.e., air flow, $\mathrm{CO} 2$ levels, humidity etc.-are the most impactful to this correlation. The importance of air quality for workplace wellbeing is also highlighted in the growing attention it has recently received in the HCI community, enhancing the importance of our early findings. Relevant work (Snow et al., 2016; Dang et al., 2020; Kim and Li, 2020; Zhong et al., 2020) addresses eco-feedback - including mobile and wearable visualizations, and ambient light notifications- and collaborative and predictive approaches to regulate air quality in office spaces (Zhong et al., 2020). However, the many elements of air quality experience in the workspace remain unaddressed by current research. Positioned within a broader sustainability agenda, our findings highlight the necessity of 'surfacing' indoor and outdoor air quality data for wellbeing in the remote/hybrid workplace; developing ambient technologies that address embodied experiences and engage the building occupants in becoming more aware and responsible in managing their micro-climate. The quantitative analysis also indicates that there are potentials of predicting core dimensions of mood based on environmental and activity data (RQ3); which will be also further explored in future work.

Although highly customizable and occupant-controlled, it appears that a level of feedback-mediated autonomy (AI) of remote workspaces would be beneficial for the occupants' wellbeing (RQ4, RQ2). This is highlighted by many of their quotes where they express getting too focused in work resulting to limited attention to their surroundings and their bodies; and the inability to constantly consider and balance all aspects of their environments (RQ4, RQ2). Moreover, participants found mood self-reporting useful, and suggested they would benefit from mood awareness strategies (RQ4). Many of them stated that they would like to be able to have a visualization of their inputs in previous days and be able to observe their behavior patterns over time. Some participants suggested the use of personalized and task - relevant feedback depending on archived data (RQ4). Regarding the predictive potentials of mood, the analysis indicated good reason to pursue verification using passively collected environmental information (RQ3). The internally coherent picture between affective data and activity indicates an adequate reliability for our exploratory findings. For many ambient working applications, it would be interesting to be able to predict affective states from passively collected data and use these as feedback towards the users, or as the basis to form rules and constraints in Human-AI interactive systems in the built environment, that help remote workers become aware of and regulate their mood (RQ4, RQ2), at an individual and collective level.

The lack of physical activity and physical movement was highly marked both in qualitative and quantitative findings (RQ2). Many participants mentioned that they do not move much during their workday as they have no physical distractions such as interruptions from colleagues and they can do everything from their desktop screens-including meetings -, resulting in reported back pain and fatigue; worsened by non-ergonomic furniture and the lack of breaks (RQ2). Wearable feedback helped in tackling some of these aspects (RQ4). Participants stated that they found the bands non-intrusive, making them move more often through display, vibration, and alarm notifications, while acknowledging their limitations in data accuracy. On-thespot wearable notifications for physical activity were successful motivators during the study, but it is unknown if this resulted in further changes in routines of the participants after the end of it. Physical activity-such as stretching breaks, cycling breaks, and particularly walking - is beneficial for physical and mental wellbeing (Haliburton and Schmidt, 2020), but it is not required or encouraged in remote work. The question is how to introduce physical movement as a core element of remote work, taking advantage of the success of wearable -mediated feedback (Gouveia et al., 2016). We suggest extending activity feedback beyond notifications, to wearable mediated ambient interactions for physical activity. 


\section{Framing a Design Agenda for Future Ambient Affective Workplaces}

Our exploratory study addressed the embodied experiences and physical aspects of the engagement with the domestic workplace. We identified some of the key challenges for the wellbeing of remote workers-such as the lack of activity and the limited or conflicting space-use. We established correlations between air quality and mood, and the importance of feedback to raise awareness on aspects of their wellbeing. Drawing on the key issues highlighted in the previous section, below we begin to sketch out a design agenda for developing ambient spaces to support the wellbeing of remote home workers. Key areas for development of ambient spaces include:

\section{1) Ambient connectivity}

Ambient connectivity between remote workspaces could support social awareness and co-presence, reducing the feeling of working in isolation; and bring the rhythmicity of the workday through ambient interactions. Ambient light, tangible media and AR projection/holographic displays could be used to provide such experiences; employing data sharing and physicalization practices to enhance the collective and informal dynamics of the workplace that are lacking in remote.

\section{2) Actuating movement}

Many mentioned that they are not walking much in remote work, as they are not required to go somewhere. We shift the discussion from providing activity feedback, to designing for wearable-mediated ambient experiences that inherently engage their occupants in a more active work life. Active ambient wok spaces could utilize wearable and embedded (furniture and textile) sensors, and soft robotic elements in furniture to lift the connected workers from their chairs and screens and support different postures and movement while working.

\section{3) Enabling Organic Transitions}

The challenges transitioning between domestic and work life and balancing different aspects of remote work life in the home have been extensively documented. Shape changing interfaces such as adaptive furniture with display capacity can support in expanding work and display areas within limited spaces, encourage mobility and posture alternation, address conflicting space uses, and support transitions between different aspects of work, and between work and domestic life.

\section{4) Passive AI comfort}

Using potentials of ambient sensory technologies, passive data processing and the diversity of smart materials; ambient workspaces could include passively intelligent features that adjust the micro-climate (particularly air quality), manage energy, and adapt to preferences and personal space-use patterns. Additionally, they could provide us with recommendations on the environmental conditions that can better support our work tasks and affective wellbeing, provide aesthetically pleasing experiences and enhance our awareness on our bodies and surroundings (eco-feedback). Addressing the experience of air quality, slow and passive environmental responses (see pneumatic elements (Fukuda et al., 2018)) could provide indications of air quality aspects and breathing rates, encouraging a more "embodied" approach to experiencing and acting on the micro-climate.

\section{5) Mood reflective architecture}

Although widely explored in past research with regards to diverse aspects of wellbeing-including arousal and stress levels (Rogers et al., 2010; Fortmann et al., 2013; Snyder et al., 2015; Pereira et al., 2016; Yu et al., 2018; Zhang et al., 2020); recent developments in programmable light (see Philips LEDs) provide new avenues for scalable experiences for mood awareness and prediction across spatiotemporal dimensions. Our quantitative results indicate that utilizing passively collected activity and ambient data to predict mood is not a farfetched idea. We propose a programmable LED system that predicts mood based on passive data, and provides feedback for awareness and personalized restoring recommendations.

\section{6) Feedback mediated autonomy}

As an overarching aspect in the discussion on ambient workspaces, we support feedback mediated autonomy (AI) of such spaces; particularly emphasizing on the potentials of wearable displays to mediate on body-environment collaboration. Ambient workspaces cannot be solely and continuously user-driven, but they should engage in a collaborative dialog with their users to develop intelligent behaviors. Ambient spaces should be privacy-friendly, utilizing passively collected data in the buildings in a transparent manner. Ambient workspaces will be foremost data -driven spaces, supporting scalability, transferability, and portability of interventions beyond the domestic and towards broader remote and hybrid workplace set ups. Ambient workspaces could have reflective and predictive capacity-reflecting trends in Human-AI interaction (Alavi et al., 2016; Alavi et al., 2020), supporting their users in gaining awareness on their mood and behaviors through utilizing data archives in the built environment and prioritizing their wellbeing over performance.

Apart from opportunities, creating ambient workspaces has many constraints and limitations; reflecting not so much on technologies but on ethics, privacy, and data accuracy in lived workspaces. As a response to ongoing concerns on privacy and the use of personal data at the workplace, this work focuses on the potentials of passively collected data only. Perceptions of privacy and agency in digital enhanced workplaces have been addressed by a previous body of work and have been considered. 


\section{Three Design Interventions for Ambient Home Workspaces}

To further illustrate how this design agenda can be made useful, we have begun to elucidate three concept designs (for future development) for ambient home workspace technologies. Each of these draws on different aspects of the design agenda above.

\section{Flex-Seat: Supporting Organic Transitions}

As highlighted in our findings, achieving the ideal home office is not easy, due to lack of space or conflicts in space use. Reflecting on the diversity in reported needs in structure, variation and flexibility, there is the broader question if a strictly "fixed" office set-up can work in a "limited, loose and flexible" environment like the domestic one. Many innovative workplaces already support hot-desking and working in lounge-format offices (see Google Zurich ${ }^{5}$ ), even active workstations (see the Edge, Amsterdam ${ }^{6}$ ). Our study of the domestic office in terms of ergonomics, reach and use zones, peripheral aspects, comfort, and positive engagement, frames the following design concepts for adaptive furniture. Enriched with sensors (such as proximity, touch, pressure, biometric), adaptive furniture can enhance the affective and physical wellbeing of remote workers through supporting different postures, predicting physical pain, and encouraging breaks. Examples of such furniture could support:

- Adaptation to your body type, posture and preferred temperature based on saved preferences (mobile app) and real time sensory data (wearable/embedded sensors), supporting ergonomic seating and comfort.

- Change of physical attributes (shape, positionality, leveling and rotation) through smart textiles and soft robotic elements to support different postures and work tasks-from creative to concentrating-and preferences.

- Connection and transferability of data between workspaces for seamless comfort.

- Actuating for physical activity through micro-movements, initiating breaks, and supporting posture alternation (between sitting and standing) depending on work tasks and preferences.

Figures 10A,B,D illustrates a concept of sensing kinetic furniture that supports posture alternation and transitioning to different work and non-work tasks. The furniture senses the ambient environment and body posture through textileembedded and wearable sensors and moves through soft robotic actuators. It syncs with your work calendar app adapting to tasks such as meetings, focus work, creative work and breaks. It allows you to design pre-plan postures and nudges you to change sitting postures with subtle movements. Figure 10

${ }^{5}$ https://www.businessinsider.com/google-zurich-headquarters-tour-2018-1? $\mathrm{r}=\mathrm{US} \& \mathrm{IR}=\mathrm{T}$

${ }^{6}$ https://www.bloomberg.com/features/2015-the-edge-the-worlds-greenest-building/

\section{Reflex-Light: Mood Predictive Architecture}

Considering the participants' interest in mood-reflective feedback in the workplace, and the rich background literature on ambient light (Rogers et al., 2010; Fortmann et al., 2013; Snyder et al., 2015; Pereira et al., 2016; Yu et al., 2018; Zhang et al., 2020); we aim to use light as mood-reflective and mood predictive strategy. Many new light products-such as Philips LEDs-enable userdriven ambient atmospheres customizable through mobile apps; creating and storing complex light scenes in different rooms, set timers and scenarios. Many of them have open APIs and embedded microphones, sync with VUIs such as Alexa and dynamically adapt to music. Utilizing open APIs of existing wearable and light devices, and processing primarily passive activity and environmental data, and secondarily voice pitch data obtained by microphones, we propose a mood-reflective/ predictive light system that supports the following:

- Dynamic adaptation to the ambient natural light of the room, support neutral light coloring or enrich with different color based on preferences; support task-relevant lighting, and turn off when away (using proximity sensors).

- Predict mood and provide feedback through temporal changes in color, intensity and rhythmicity of the light to help users become aware of changes in their mood, and help them restore based on preferences.

- Enhancing social awareness and group wellbeing, connecting remote workspaces through sharing aggregated mood data through light infrastructure.

- Control via proximity or touch sensors and wearable sensors.

Figures 11A,B illustrates a prototype set up including Philips Hue and Mbient ${ }^{7}$ sensors (ambient data), using light to reflect and predict mood. Figures 11C,D illustrate experiences in mood reflective spaces. Future work will explore spatiotemporal aspects of light as feedback-i.e., changes in color, rhythmicity, brightness, intensity, scale, synchronous and asynchronous change- in remote workplace with regards to mood awareness.

\section{Air Flow: Bio-Design for Air Quality}

It derives from the participants' responses that as they focus on their work, they cannot always be aware of all aspects of their surroundings. The study gave them opportunities to evaluate aspects of their micro-climate-such as felt temperature and perceived air quality and reflect on their mood. Air quality gave an interesting correlation to valence (positive-negative mood) in quantitative data, being non-present as a documented experience in the qualitative data. Therefore, "surfacing" air quality data to increase the remote workers awareness on air quality could have a positive impact on their wellbeing and mood.

There are many aspects of air quality experience and management unaddressed by current research on the workplace (Snow et al., 2016; Dang et al., 2020; Kim and Li, 2020; Zhong et al., 2020). The problem of poor indoor air quality is often difficult to solve as air can be more polluted outside, and

\footnotetext{
${ }^{7}$ See mbientLab https://mbientlab.com/
} 

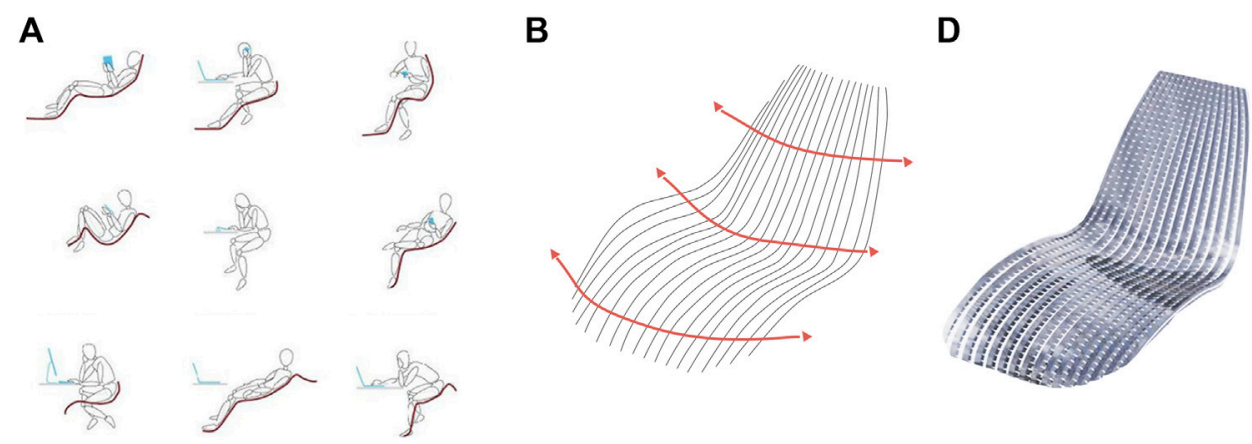

FIGURE 10 | Flex-seat: a sensing adaptive kinetic chair (concept) for posture management. Image source for (A): https://www.behance.net/gallery/16827089/ Parametric-Waffle-Bench.

A

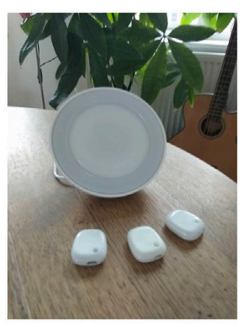

C

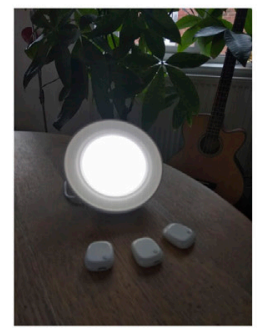

B
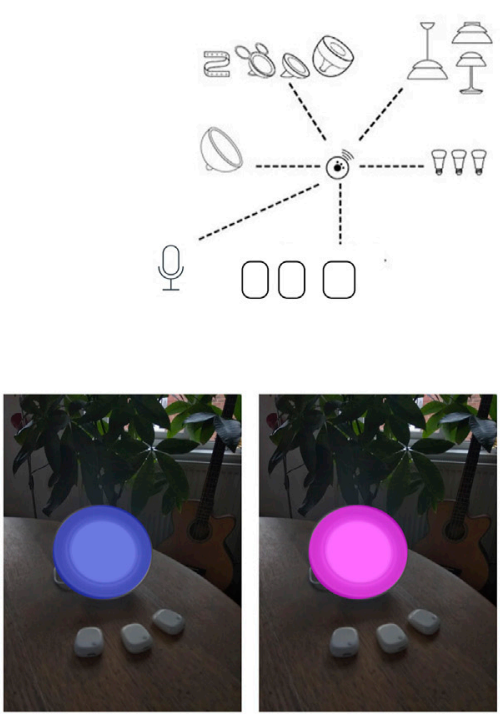

D
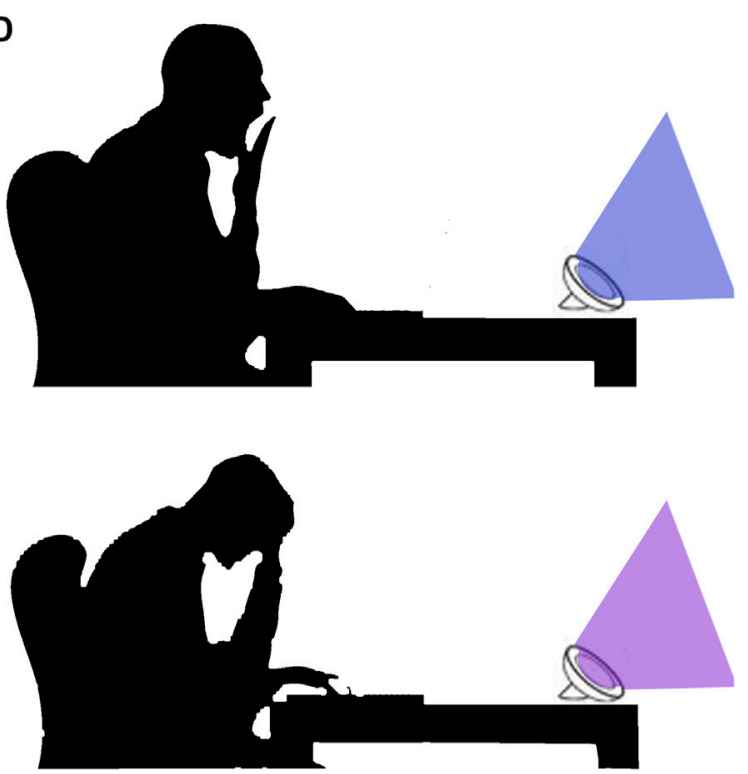

FIGURE 11 | Reflex-light: mood predictive architecture.

A

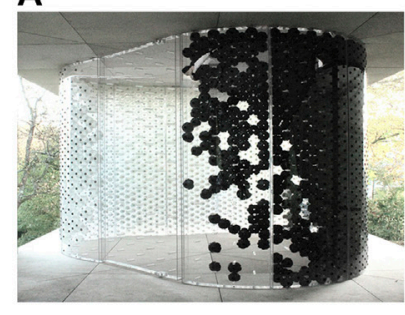

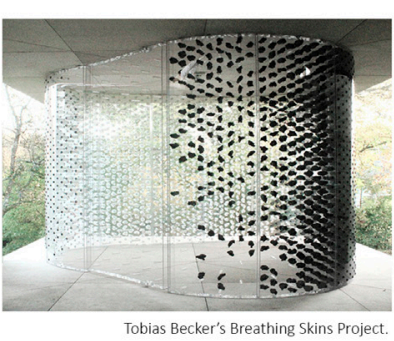

B

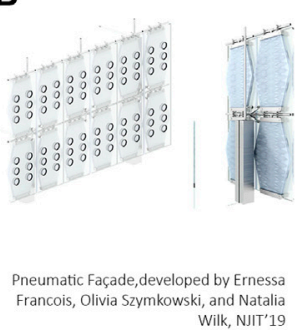

ilk, NIIT'19

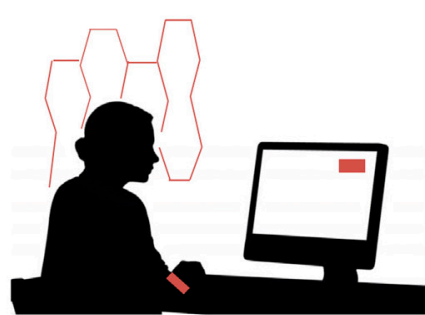

FIGURE 12 | Air flow: bio-design for air quality. Image source for (A): Tobias Becker's Breathing Skins Project (https://www.archdaily.com/789230/let-yourbuilding-to-breathe-with-this-pneumatic-facade-technology). Image source for (B): Pneumatic Façade: The project was developed by Ernessa Francois, Olivia Szymkowski, and Natalia Wilk, NJIT'19 for the Adaptive and Autonomous Environments Options Studio III, advised by professor Andrzej Zarzycki, NJIT (http://andrzejzarzycki.com/pneumatic-facade/).

opening a window can mean increasing noise or decreasing heating. Additionally, much of the research on air quality is limited to $\mathrm{CO} 2$, leaving humidity, air temperature and air flow aside. Further questions include what types of ambient feedback might better address users' experiences of air quality, bringing sensory readings closer to the embodied experiences of the 
occupants. Physical representations of air quality feedback that are closer to the embodied experience could deepen awareness of one's body and environment, and how these two aspects relate to each other.

Bringing features from adaptive architecture (Yannoudes, 2016; Eltaweel and SU, 2017), modular features of breathing skins such as pneumatic surfaces (Fukuda et al., 2018) (Figure 12A) installed in the periphery could support a more embodied awareness of microclimate and comfort, whilst improving indoor air circulation and providing a visually aesthetic experience. Such elements could be enhanced with visualizations of ambient air quality data or synchronized with breathing rate of occupants. Figure 12B is a breathing surface concept; an aesthetic visual barrier coordinating with breathing rate through a wearable, assisting air circulation and breathing regulation, and providing occupants with information of air quality at their desktop.

\section{Limitations}

This study had a number of limitations. We acknowledge that the sample for the quantitative study is relatively small and that these early findings are indicative. Quantitative findings will be validated in future work with a larger number of participants and repeated measurements using sensory readings, and not solely self-reported data. The proposed design concepts that address wellbeing aspects in the built environment are in concept stage. Besides validating findings, future work will focus on developing working prototypes of the design concepts addressed, evaluate these with users in lived-in remote home workplaces, and evaluate and discuss the impact on wellbeing following a mixed methods approach.

\section{CONCLUSION}

This work addresses the wellbeing challenges for remote home workers during the pandemic and provides insights on their engagement with the domestic workplace. Through a mixedmethods approach, this study establishes correlations between mood and physical aspects of the domestic workplace and discusses the impact of feedback mechanisms (wearables and self-reporting) on the behavior of remote workers. Key findings were the actions to maximize comfort addressing visual, auditory and kinesthetic aspects; the lack of physical activity, the lack of workday rhythmicity (i.e., spatial variation and social interactions), and a latent correlation between valence and perceived air quality. These findings led to a framing of an "ambient workspaces" design agenda to support the wellbeing of the remote home workers through feedback; designing ambient spaces that support embodied

\section{REFERENCES}

Alavi, H. S., Churchill, E., Kirk, D., Bier, H., Verma, H., Lalanne, D., et al. (2018). From Artifacts to Architecture. In Proceedings of the 2018 ACM Conference Companion Publication on Designing Interactive Systems (DIS'18 Companion). Association for Computing Machinery. Hong Kong, China. New York, NY: ACM Press, 387-390. doi:10.1145/3197391.3197393 and environmental awareness through passive sensing. We further presented three concept projects as illustrations of how this design agenda can be used to further develop ambient workspaces that support different and broader aspects of wellbeing through feedback. The proposed agenda is composed under the premise of co-creating more intelligent environments together with their occupants; this is useful to guide future interventions in the context of remote and hybrid workplaces and highlights areas of critical research interest for Human-Building-Interaction and Human-AI Interaction in the Built Environment. Future work will expand on validating correlations, developing working prototypes based on the discussed agenda and will evaluate the impact of the interventions on the wellbeing and behavior of remote home workers.

\section{DATA AVAILABILITY STATEMENT}

The raw data supporting the conclusions of this article will be made available by the authors, without undue reservation.

\section{ETHICS STATEMENT}

Ethical review and approval was not required for the study on human participants in accordance with the local legislation and institutional requirements. The patients/participants provided their written informed consent to participate in this study.

\section{AUTHOR CONTRIBUTIONS}

EM led the work, developed the study design, collected and analysed the data and drafted the paper. RA contributed to the quantitative analysis, arranged and processed the data; developed statistical and supervised learning methods. DV contributed to formulating the literature review. RB assisted with advancing the quantitative analysis. JS assisted with study design, contributed to the analysis of the quant data and edited the manuscript. DK assisted with study design, supervised and directed the research, contributed to the analysis of the qual data and edited the manuscript.

\section{ACKNOWLEDGMENTS}

This work was partially funded by the EPSRC (EP/M023001/1).

Alavi, H. S., Churchill, E., Kirk, D., Nembrini, J., and Lalanne, D. (2016) Deconstructing Human-Building Interaction. interactions 23, 60-62. doi:10.1145/2991897

Alavi, H. S., Lalanne, D., and Rogers, Y. (2020). The Five Strands of Living Lab. ACM Trans. Comput.-Hum. Interact. 27, 1-26. doi:10.1145/3380958

Alavi, H. S., Verma, H., Mlynar, J., and Lalanne, D. (2018). The Hide and Seek of Workspace: Towards Human-Centric Sustainable Architecture. Proceedings of the 2018 CHI Conference on Human Factors in Computing Systems. 
Association for Computing Machinery. Montreal QC, Canada. New York, NY: ACM Press, 1-12. doi:10.1145/3173574.3173649

Alavi, H. S., Verma, H., Papinutto, M., and Lalanne, D. (2017). "Comfort: A Coordinate of User Experience in Interactive Built Environments," in HumanComputer Interaction - INTERACT 2017. Editors R. Bernhaupt, G. Dalvi, A. Joshi, K. D. Balkrishan, J. O'Neill, and M. Winckler (Cham: Springer International Publishing), 247-257. doi:10.1007/978-3-319-67687-6_16

Alonso, M. B., Keyson, D. V., and Hummels, C. C. M. (2008). Squeeze, Rock, and Roll; Can Tangible Interaction with Affective Products Support Stress Reduction? Proceedings of the 2nd International Conference on Tangible and Embedded Interaction - TEI '08. Presented at the the 2nd international conference. Bonn, Germany. New York, NY: ACM Press, 105. doi:10.1145/ 1347390.1347413

Aryal, A., Anselmo, F., and Becerik-Gerber, B. (2018). Smart IoT Desk for Personalizing Indoor Environmental Conditions. Proceedings of the 8th International Conference on the Internet of Things. Presented at the IOT '18: 8th International Conference on the Internet of Things. Santa Barbara California USA. New York, NY: ACM, 1-6. doi:10.1145/3277593.3277614

Bader, P., Voit, A., Le, H. V., Woźniak, P. W., Henze, N., and Schmidt, A. (2019). WindowWall. ACM Trans. Comput.-Hum. Interact. 26, 1-42. doi:10.1145/ 3310275

Bakker, S., Hausen, D., and Selker, T. (2016). Peripheral Interaction. New York, NY: Springer Berlin Heidelberg.

Betella, A., and Verschure, P. F. M. J. (2016). The Affective Slider: A Digital SelfAssessment Scale for the Measurement of Human Emotions. PLoS ONE 11, e0148037. doi:10.1371/journal.pone.0148037

Bittner, B., Aslan, I., Dang, C. T., and André, E. (2019). Of Smarthomes, IoT Plants, and Implicit Interaction Design. Proceedings of the Thirteenth International Conference on Tangible, Embedded, and Embodied Interaction. Presented at the TEI '19: Thirteenth International Conference on Tangible, Embedded, and Embodied Interaction. Tempe Arizona USA. New York, NY: ACM, 145-154. doi:10.1145/3294109.3295618

Boehner, K., DePaula, R., Dourish, P., and Sengers, P. (2005). Affect. Proceedings of the 4th Decennial Conference on Critical Computing between Sense and Sensibility - CC '05. Presented at the the 4th decennial conference. Aarhus, Denmark. New York, NY: ACM Press, 59. doi:10.1145/1094562.1094570

Brombacher, H., Ren, X., Vos, S., and Lallemand, C. (2020). Visualizing ComputerBased Activity on Ambient Displays to Reduce Sedentary Behavior at Work 5. doi:10.1145/3441000.3441022

Cambo, S. A., Avrahami, D., and Lee, M. L. (2017). BreakSense. Proceedings of the 2017 CHI Conference on Human Factors in Computing Systems. Presented at the CHI '17: CHI Conference on Human Factors in Computing Systems. Denver Colorado USA. New York, NY: ACM, 3595-3607. doi:10.1145/ 3025453.3026021

Campos, P. F., Cabral, D., and Gonçalves, F. (2018). Sense.Seat. The 31st Annual ACM Symposium on User Interface Software and Technology Adjunct Proceedings. Presented at the UIST '18: The 31st Annual ACM Symposium on User Interface Software and Technology. Berlin, Germany, New York, NY: ACM, 72-74. doi:10.1145/3266037.3266105

Caraban, A., Karapanos, E., Gonçalves, D., and Campos, P. (2019). 23 Ways to Nudge. Proceedings of the 2019 CHI Conference on Human Factors in Computing Systems - CHI '19. Presented at the the 2019 CHI Conference. Glasgow, Scotland Uk. New York, NY: ACM Press, 1-15. doi:10.1145/ 3290605.3300733

Chodan, W., Krause, S., Meza-Cuevas, M. A., Kadner, M., Rockstroh, J., König, C., et al. (2019). The SEBA System. Proceedings of the 6th International Workshop on Sensor-Based Activity Recognition and Interaction. Presented at the iWOAR '19: 6th international Workshop on Sensor-based Activity Recognition and Interaction. Rostock Germany. New York, NY: ACM, 1-6. doi:10.1145/3361684.3361694

Choi, W., Song, A., Edge, D., Fukumoto, M., and Lee, U. (2016). Exploring User Experiences of Active Workstations. Proceedings of the 2016 ACM International Joint Conference on Pervasive and Ubiquitous Computing. Presented at the UbiComp '16: The 2016 ACM International Joint Conference on Pervasive and Ubiquitous Computing. Heidelberg Germany. New York, NY: ACM, 805-816. doi:10.1145/2971648.2971756

Church, K., Hoggan, E., and Oliver, N. (2010). A Study of mobile Mood Awareness and Communication through MobiMood. Proceedings of the 6th Nordic
Conference on Human-Computer Interaction Extending Boundaries NordiCHI '10. Presented at the the 6th Nordic Conference. Reykjavik, Iceland. New York, NY: ACM Press, 128. doi:10.1145/1868914.1868933

Clear, A. K., Mitchell Finnigan, S., Olivier, P., and Comber, R. (2018). ThermoKiosk. Proceedings of the $2018 \mathrm{CHI}$ Conference on Human Factors in Computing Systems - CHI '18. Presented at the the 2018 CHI Conference. Montreal QC, Canada. New York, NY: ACM Press, 1-12. doi:10.1145/3173574.3173956

Constantinides, M., Šćepanović, S., Quercia, D., Li, H., Sassi, U., and Eggleston, M. (2020). ComFeel. Proc. ACM Interact. Mob. Wearable Ubiquitous Technol. 4, 1-21. doi:10.1145/3432234

Dalton, N. S. (2016). Architecture and Interaction: Human Computer Interaction in Space and Place. New York, NY: Springer Berlin Heidelberg.

Damen, I., Lallemand, C., Brankaert, R., Brombacher, A., van Wesemael, P., and Vos, S. (2020). Understanding Walking Meetings: Drivers and Barriers. Proceedings of the $2020 \mathrm{CHI}$ Conference on Human Factors in Computing Systems. Presented at the CHI '20: CHI Conference on Human Factors in Computing Systems. Honolulu HI USA. New York, NY: ACM, 1-14. doi:10.1145/3313831.3376141

Dang, C. T., Aslan, I., Seiderer, A., and André, E. (2020). Exploring Ambient Air Quality Notifications for Smart Rooms. Proceedings of the 6th EAI International Conference on Smart Objects and Technologies for Social Good. Presented at the GoodTechs '20: 6th EAI International Conference on Smart Objects and Technologies for Social Good. Antwerp Belgium. New York, NY: ACM, 102-107. doi:10.1145/3411170.3411229

Davis, K., Feijs, L., Hu, J., Marcenaro, L., and Regazzoni, C. (2016). Improving Awareness and Social Connectedness through the Social Hue. Proceedings of the International Symposium on Interactive Technology and Ageing Populations - ITAP '16. Presented at the the International Symposium. Kochi, Japan. New York, NY: ACM Press, 12-23. doi:10.1145/2996267.2996269

Deloitte LLP (2018). The Connected Worker. Availableat: https://www2.deloitte. $\mathrm{com} / \mathrm{uk} / \mathrm{en} / \mathrm{pages} / \mathrm{consumer}$-industrial-products/articles/the-connectedworker.html.

Elsden, C., Selby, M., Durrant, A., and Kirk, D. (2016). Fitter, Happier, More Productive: What to Ask of a Data-Driven Life. Interactions 5.

Eltaweel, A., and Su, Y. (2017). Parametric Design and Daylighting: A Literature Review. Renew. Sustainable Energ. Rev. 73, 1086-1103. doi:10.1016/ j.rser.2017.02.011

Epstein, D. A., Avrahami, D., and Biehl, J. T. (2016). Taking 5. Proceedings of the 2016 CHI Conference on Human Factors in Computing Systems. Presented at the CHI'16: CHI Conference on Human Factors in Computing Systems. San Jose California USA. New York, NY: ACM, 673-684. doi:10.1145/2858036.2858066

Fan Ng, C. (2010). Teleworker's home Office: an Extension of Corporate Office? Facilities 28 (3/4), 137-155. doi:10.1108/02632771011023113

Feurer, M., Eggensperger, K., Falkner, S., Lindauer, M., and Hutter, F. (2020). Auto-Sklearn 2.0: The Next Generation. ArXiv:2007.04074 [Cs, Stat]. Available at: http://arxiv.org/abs/2007.04074.

Follmer, S., Leithinger, D., Olwal, A., Hogge, A., and Ishii, H. (2013). inFORM. Proceedings of the 26th Annual ACM Symposium on User Interface Software and Technology (UIST '13). New York, NY, USA: Association for Computing Machinery, 417-426. doi:10.1145/2501988.2502032

Fortmann, J., Stratmann, T., Boll, S., Poppinga, B., and Heuten, W. (2013). Make Me Move at Work! an Ambient Light Display to Increase Physical Activity. Proceedings of the 7th International Conference on Pervasive Computing Technologies for Healthcare (PervasiveHealth '13). Brussels, BEL: ICST, 274-277. doi:10.4108/icst.pervasivehealth.2013.252089

Gouveia, R., Pereira, F., Karapanos, E., Munson, S. A., and Hassenzahl, M. (2016). Exploring the Design Space of Glanceable Feedback for Physical Activity Trackers. Proceedings of the 2016 ACM International Joint Conference on Pervasive and Ubiquitous Computing. Presented at the UbiComp '16: The 2016 ACM International Joint Conference on Pervasive and Ubiquitous Computing. Heidelberg Germany. New York, NY: ACM, 144-155. doi:10.1145/ 2971648.2971754

Grønbæk, J. E., Korsgaard, H., Petersen, M. G., Birk, M. H., and Krogh, P. G. (2017). Proxemic Transitions: Designing Shape-Changing Furniture for Informal Meetings. Proceedings of the 2017 CHI Conference on Human Factors in Computing Systems. Presented at the CHI '17: CHI Conference on Human Factors in Computing Systems. Denver Colorado USA. New York, NY: ACM, 7029-7041. doi:10.1145/3025453.3025487 
Grønbæk, J. E., Rasmussen, M. K., Halskov, K., and Petersen, M. G. (2020). KirigamiTable: Designing for Proxemic Transitions with a Shape-Changing Tabletop. Proceedings of the 2020 CHI Conference on Human Factors in Computing Systems. Presented at the CHI '20: CHI Conference on Human Factors in Computing Systems. Honolulu HI USA. New York, NY: ACM, 1-15. doi:10.1145/3313831.3376834

Haliburton, L., and Schmidt, A. (2020). Technologies for Healthy Work. Interactions 27, 64-66. doi:10.1145/3386391

Hänsel, K. (2016). Wearable and Ambient Sensing for Well-Being and Emotional Awareness in the Smart Workplace. Proceedings of the 2016 ACM International Joint Conference on Pervasive and Ubiquitous Computing: Adjunct. Presented at the UbiComp '16: The 2016 ACM International Joint Conference on Pervasive and Ubiquitous Computing. Heidelberg Germany. New York, NY: ACM, 411-416. doi:10.1145/ 2968219.2971360

Hossain, N., and Nazin, M. (2018). EmoVoice. Proceedings of the 2018 ACM International Joint Conference and 2018 International Symposium on Pervasive and Ubiquitous Computing and Wearable Computers. Presented at the UbiComp '18: The 2018 ACM International Joint Conference on Pervasive and Ubiquitous Computing. Singapore Singapore. New York, NY: ACM, 1826-1828. doi:10.1145/3267305.3277832

Huber, J. (2014). A Research Overview of Mobile Projected User Interfaces. Informatik Spektrum 37, 464-473. doi:10.1007/s00287-014-0819-z

Jäger, N. (2017). Interacting with Adaptive Architecture. Interactions 24, 62-65. doi:10.1145/3137113

Jäger, N., Schnädelbach, H., Hale, J., Kirk, D., and Glover, K. (2017). Reciprocal Control in Adaptive Environments. Interact. Comput. iww037v1. doi:10.1093/ iwc/iww037

Jonathan, Z. B., and Laura, R. M. (2017). Repeated measures correlation. Front. Psychol. doi:10.3389/fpsyg.2017.00456

Katabi, D., Adib, F., Mao, H., Kabelac, Z., and Miller, R. C. (2015). Smart Homes that Monitor Breathing and Heart Rate. Proceedings of the 33rd Annual ACM Conference on Human Factors in Computing Systems - CHI '15. Presented at the the 33rd Annual ACM Conference. Seoul, Republic of Korea. New York, NY: ACM Press, 837-846. doi:10.1145/2702123.2702200

Khazan, O. (2020). Work from Home Is Here to Stay. The Atlantic. Avaliable at: https://www.theatlantic.com/health/archive/2020/05/work-from-home-pandemic/ 611098/Last (Accessed 02 27, 2021).

Kikhia, B., and Hallberg, J. (2013). Visualizing and Managing Stress through Colors and Images. Proceedings of the 4th International Sense Cam \& Pervasive Imaging Conference on - SenseCam '13. Presented at the the 4th International SenseCam \& Pervasive Imaging Conference. San Diego, California. New York, NY: ACM Press, 78-79. doi:10.1145/2526667.2526680

Kim, S., and Li, M. (2020). Awareness, Understanding, and Action: A Conceptual Framework of User Experiences and Expectations about Indoor Air Quality Visualizations. Proceedings of the 2020 CHI Conference on Human Factors in Computing Systems. Presented at the CHI '20: CHI Conference on Human Factors in Computing Systems. Honolulu HI USA. New York, NY: ACM, 1-12. doi: $10.1145 / 3313831.3376521$

Lee, B., Lee, M., Zhang, P., Tessier, A., Saakes, D., and Khan, A. (2021). SocioSpatial Comfort. Proc. ACM Hum.-Comput. Interact. 4, 1-33. 10.1145/3432937.

Lee, H., Je, S., Kim, R., Verma, H., Alavi, H., and Bianchi, A. (2019). Partitioning Open-Plan Workspaces via Augmented Reality. Pers Ubiquit Comput. doi:10.1007/s00779-019-01306-0

Ludden, G. D. S., and Meekhof, L. (2016). Slowing Down. Proceedings of the 28th Australian Conference on Computer-Human Interaction (OzCHI '16). New York, NY, USA: Association for Computing Machinery, 435-441. doi:10.1145/ 3010915.3010938

Mark, G., Iqbal, S., Czerwinski, M., and Johns, P. (2014). Capturing the Mood. Proceedings of the 17th ACM Conference on Computer Supported Cooperative Work \& Social Computing - CSCW '14. Presented at the the 17th ACM conference. Baltimore, Maryland, USA. New York, NY: ACM Press, 1082-1094. doi:10.1145/2531602.2531673

Mark, G., Iqbal, S., Czerwinski, M., and Johns, P. (2015). Focused, Aroused, but So Distractible. Proceedings of the 18th ACM Conference on Computer Supported Cooperative Work \& Social Computing - CSCW '15. Presented at the the 18th ACM Conference. Vancouver, BC, Canada. New York, NY: ACM Press, 903-916. doi:10.1145/2675133.2675221
Meyer, A. N., Murphy, G. C., Zimmermann, T., and Fritz, T. (2017). Design Recommendations for Self-Monitoring in the Workplace. Proc. ACM Hum.Comput. Interact. 1, 1-24. doi:10.1145/3134714

Mishra, S. R., and Klasnja, P. (2017). "Move into Another World of Happy". Proceedings of the 11th EAI International Conference on Pervasive Computing Technologies for Healthcare (PervasiveHealth '17). New York, NY, USA: Association for Computing Machinery, 21-30. doi:10.1145/ 3154862.3154880

Mora, S., Rivera-Pelayo, V., and Mueller, L. (2011). Supporting Mood Awareness in Collaborative Settings. Proceedings of the 7th International Conference on Collaborative Computing: Networking, Applications and Worksharing. Presented at the 7th International Conference on Collaborative Computing: Networking, Applications and Worksharing. Orlando, United States: IEEE. doi:10.4108/icst.collaboratecom.2011.247091

Nabil, S., Kirk, D. S., Ploetz, T., and Wright, P. (2017). Designing Future Ubiquitous Homes with OUI Interiors: Possibilities and Challenges 12. doi:10.1145/ 3064663.3064745

Nabil, S., Plötz, T., and Kirk, D. S. (2017). Interactive Architecture. Proceedings of the Tenth International Conference on Tangible, Embedded, and Embodied Interaction - TEI '17. Presented at the the Tenth International Conference. Yokohama, Japan. New York, NY: ACM Press, 89-100. doi:10.1145/ 3024969.3024981

Nembrini, J., and Lalanne, D. (2017). "Human-Building Interaction: When the Machine Becomes a Building," in Human-Computer Interaction - INTERACT 2017. Editors R. Bernhaupt, G. Dalvi, A. Joshi, K. D. Balkrishan, J. O’Neill, and M. Winckler (Cham: Springer International Publishing), 348-369. doi:10.1007/ 978-3-319-67684-5_21

Nowell, L. S., Norris, J. M., White, D. E., and Moules, N. J. (2017). Thematic Analysis. Int. J. Qual. Methods 16, 160940691773384. doi:10.1177/1609406917733847

Pereira, E., Kolb, S., Schäfers, K., Schreiber, S., Weckbach, V., Goodwin, W., et al. (2016). Stars. Proceedings of the 2016 ACM International Joint Conference on Pervasive and Ubiquitous Computing: Adjunct. Presented at the UbiComp '16: The 2016 ACM International Joint Conference on Pervasive and Ubiquitous Computing. Heidelberg Germany. New York, NY: ACM, 1640-1645. doi:10.1145/2968219.2968527

Perteneder, F., Grossauer, E.-M. B., Leong, J., Stuerzlinger, W., and Haller, M. (2016). Glowworms and Fireflies. Proceedings of the 2016 CHI Conference on Human Factors in Computing Systems. Presented at the CHI'16: CHI Conference on Human Factors in Computing Systems. San Jose California USA. New York, NY: ACM, 5849-5861. doi:10.1145/2858036.2858524

Petersen, M. G., Rasmussen, M. K., and Trettvik, J. (2020). Affordances of ShapeChanging Interfaces. Proceedings of the 2020 ACM Designing Interactive Systems Conference. Presented at the DIS '20: Designing Interactive Systems Conference 2020. Eindhoven Netherlands. New York, NY: ACM, 1959-1971. doi:10.1145/3357236.3395521

Petford, J., Nacenta, M. A., Gutwin, C., Eremondi, J., and Ede, C. (2016). The ASPECTA Toolkit. Proceedings of the 5th ACM International Symposium on Pervasive Displays. Presented at the PerDis '16: The International Symposium on Pervasive Displays. Oulu Finland. New York, NY: ACM, 87-105. doi:10.1145/2914920.2915006

Pousman, Z., and Stasko, J. (2006). A Taxonomy of Ambient Information Systems. Proceedings of the working conference on Advanced visual interfaces (AVI'06). New York, NY, USA: Association for Computing Machinery, 67-74. doi:10.1145/1133265.1133277

Rogers, Y., Hazlewood, W. R., Marshall, P., Dalton, N., and Hertrich, S. (2010). Ambient Influence. Proceedings of the 12th ACM International Conference on Ubiquitous Computing. Presented at the Ubicomp '10: The 2010 ACM Conference on Ubiquitous Computing. Copenhagen Denmark. New York, NY: ACM, 261-270. doi:10.1145/1864349.1864372

Rosenberg, D., and Tsamis, A. (2019). Human-Building Collaboration. Proceedings of the 24th International Conference of the Association for Computer-Aided Architectural Design Research in Asia, 2. Hong Kong: CAADRIA, 171-180.

Russell, J. A. (1980). A circumplex model of affect. . Journal of Personality and Social Psychology 39(6), 1161-1178. doi:10.1037/h0077714

Sanches, P., Janson, A., Karpashevich, P., Nadal, C., Qu, C., Daudén Roquet, C., et al. (2019). HCI and Affective Health. Proceedings of the $2019 \mathrm{CHI}$ Conference on Human Factors in Computing Systems - CHI '19. Presented 
at the the 2019 CHI Conference. Glasgow, Scotland Uk. New York, NY: ACM Press, 1-17. doi:10.1145/3290605.3300475

Schimmack, U., and Grob, A. (2000). Dimensional models of core affect: A quantitative comparison by means of structural equation modeling. European Journal of Personality 14(4), 325-345. doi:10.1002/10990984(200007/08)3.0.CO;2-I

Schnädelbach, H., Irune, A., Kirk, D., Glover, K., and Brundell, P. (2012). ExoBuilding. ACM Trans. Comput.-Hum. Interact. 19, 1-22. doi:10.1145/ 2395131.2395132

Schnädelbach, H., and Kirk, D. (2019). People, Personal Data and the Built Environment. 1st. Incorporated: Springer Publishing Company.

Snow, S., Auffenberg, F., and schraefel, m. c. (2017). Log it while It's Hot. Proceedings of the 2017 CHI Conference on Human Factors in Computing Systems. Presented at the CHI '17: CHI Conference on Human Factors in Computing Systems. Denver Colorado USA. New York, NY: ACM, 1595-1606. doi:10.1145/3025453.3025578

Snow, S., Soska, A., Chatterjee, S. K., and schraefel, m. c. (2016). Keep Calm and Carry on. Proceedings of the 2016 CHI Conference Extended Abstracts on Human Factors in Computing Systems. Presented at the CHI'16: CHI Conference on Human Factors in Computing Systems. San Jose California USA. New York, NY: ACM, 1476-1482. doi:10.1145/2851581.2892490

Snyder, J., Matthews, M., Chien, J., Chang, P. F., Sun, E., Abdullah, S., and Gay, G. (2015). "MoodLight: Exploring Personal and Social Implications of Ambient Display of Biosensor Data," in Proceedings of the 18th ACM Conference on Computer Supported Cooperative Work \& Social Computing (CSCW '15), New York, NY, USA (New York, NY: Association for Computing Machinery), 143-153. doi:10.1145/ 2675133.2675191

Stawarz, K., Cox, A. L., Bird, J., and Benedyk, R. (2013). "I'd Sit at home and Do Work Emails". CHI '13 Extended Abstracts on Human Factors in Computing Systems (CHI EA '13). New York, NY, USA. New York, NY: Association for Computing Machinery, 1383-1388. doi:10.1145/2468356.2468603

Takashima, K., Oyama, T., Asari, Y., Sharlin, E., Greenberg, S., and Kitamura, Y. (2016). Study and Design of a Shape-Shifting Wall Display. Proceedings of the 2016 ACM Conference on Designing Interactive Systems. Presented at the DIS '16: Designing Interactive Systems Conference 2016. New York, NY: ACM,Brisbane QLD Australia, 796-806. doi:10.1145/ 2901790.2901892

T. Fukuda, W. Huang, P. Janssen, K. Crolla, and S. Alhadidi, (2018). "Learning, Adapting and Prototyping,"Proceedings of the 23rd International Conference of the Association for Computer-Aided Architectural, Design Research in Asia (CAADRIA). Hong Kong. 2, 143-151.

Toch, E., Chassidim, H., and Hatuka, T. (2020). Can You Turn it off?. Proc. ACM Hum.-Comput. Interact. 4, 1-18. doi:10.1145/3415162

Weiser, M., and Brown, J. S., 1995. Designing Calm Technology. Xerox PARC 5.
Winkler, C., Seifert, J., Dobbelstein, D., and Rukzio, E. (2014). Pervasive Information through Constant Personal Projection. Proceedings of the SIGCHI Conference on Human Factors in Computing Systems. Presented at the CHI '14: CHI Conference on Human Factors in Computing Systems. Toronto Ontario Canada. New York, NY: ACM, 4117-4126. doi:10.1145/ 2556288.2557365

Wohn, D. Y., Kum-Biocca, H. H., Sharma, A., and Khandakar, A. (2020). A Room with a "Fake" View: Installing Digital Windows in Windowless Offices. ACM International Conference on Interactive Media Experiences. Presented at the IMX '20: ACM International Conference on Interactive Media Experiences, ACM. Cornella, Barcelona Spain, 180-184. doi:10.1145/3391614.3399397

Yannoudes, S. (2016). Architecture and Adaptation: From Cybernetics to Tangible Computing. New York, NY: Routledge.

Yu, B., Hu, J., Funk, M., and Feijs, L. (2018). DeLight: Biofeedback through Ambient Light for Stress Intervention and Relaxation Assistance. Pers Ubiquit Comput. 22, 787-805. doi:10.1007/s00779-018-1141-6

Zhang, D., Park, J. W., Zhang, Y., Zhao, Y., Wang, Y., Li, Y., et al. (2020). OptoSense. Proc. ACM Interact. Mob. Wearable Ubiquitous Technol. 4, 1-27. doi: $10.1145 / 3411826$

Zhao, N., Azaria, A., and Paradiso, J. A. (2017). Mediated Atmospheres. Proc. ACM Interact. Mob. Wearable Ubiquitous Technol. 1, 1-23. doi:10.1145/ 3090096

Zhong, S., Alavi, H. S., and Lalanne, D. (2020). Hilo-wear: Exploring Wearable Interaction with Indoor Air Quality Forecast. Extended Abstracts of the 2020 CHI Conference on Human Factors in Computing Systems. Presented at the CHI '20: CHI Conference on Human Factors in Computing Systems. Honolulu HI USA. New York, NY: ACM, 1-8. doi:10.1145/3334480.3382813

Conflict of Interest: The authors declare that the research was conducted in the absence of any commercial or financial relationships that could be construed as a potential conflict of interest.

Publisher's Note: All claims expressed in this article are solely those of the authors and do not necessarily represent those of their affiliated organizations, or those of the publisher, the editors and the reviewers. Any product that may be evaluated in this article, or claim that may be made by its manufacturer, is not guaranteed or endorsed by the publisher.

Copyright () 2021 Margariti, Ali, Benthem de Grave, Verweij, Smeddinck and Kirk. This is an open-access article distributed under the terms of the Creative Commons Attribution License (CC BY). The use, distribution or reproduction in other forums is permitted, provided the original author(s) and the copyright owner(s) are credited and that the original publication in this journal is cited, in accordance with accepted academic practice. No use, distribution or reproduction is permitted which does not comply with these terms. 